\title{
LIE ALGEBRAS SIMPLE WITH RESPECT TO A TAFT ALGEBRA ACTION
}

\author{
A. S. GORDIENKO
}

\begin{abstract}
We classify finite dimensional $H_{m^{2}}(\zeta)$-simple $H_{m^{2}}(\zeta)$-module Lie algebras $L$ over an algebraically closed field of characteristic 0 where $H_{m^{2}}(\zeta)$ is the $m$ th Taft algebra. As an application, we show that despite the fact that $L$ can be non-semisimple in ordinary sense, $\lim _{n \rightarrow \infty} \sqrt[n]{c_{n}^{H_{m^{2}}(\zeta)}(L)}=\operatorname{dim} L$ where $c_{n}^{H_{m^{2}}(\zeta)}(L)$ is the codimension sequence of polynomial $H_{m^{2}}(\zeta)$-identities of $L$. In particular, the analog of Amitsur's conjecture holds for $c_{n}^{H_{m^{2}}(\zeta)}(L)$.
\end{abstract}

\section{INTRODUCTION}

An $H_{m^{2}}(\zeta)$-module algebra, where $\zeta$ is a primitive $m$ th root of unity, $m \in \mathbb{N}, m \geqslant 2$, is an algebra $A$ endowed with an automorphism $c$ and a skew-derivation $v$ such that $v c=\zeta c v$, $c^{m}=\mathrm{id}_{A}$, and $v^{m}=0$. In particular, $A$ is a $\mathbb{Z}_{m}$-graded algebra where $\mathbb{Z}_{m}=\mathbb{Z} / m \mathbb{Z}$ is the cyclic group of order $m$ (see Remark 2.3). $H_{m^{2}}(\zeta)$-module algebras provide, probably, easiest examples of $H$-module algebras for a non-semisimple Hopf algebra $H$. The study of $H$-module algebras can be considered as the next logical step after the investigation of graded algebras, which have been studied extensively (see e.g. [2, 5, 23]). In the context of polynomial identities, $H$-module Lie algebras were considered in [18]. It is worth to notice that $H_{m^{2}}(\zeta)$-module algebras can have a structure quite different from the structure of $H$ module algebras for a semisimple Hopf algebra $H$. For example, an $H_{m^{2}}(\zeta)$-simple (i.e. not containing non-trivial $H_{m^{2}}(\zeta)$-invariant ideals) algebra can have a non-trivial radical.

Finite dimensional associative $H_{m^{2}}(\zeta)$-module algebras that contain no nonzero nilpotent elements were classified in [22, Theorem 2.5]. Exact module categories over the category $\operatorname{Rep}\left(H_{m^{2}}(\zeta)\right)$ were studied in [6, Theorem 4.10]. Finite dimensional associative $H_{m^{2}}(\zeta)$ simple $H_{m^{2}}(\zeta)$-module algebras were classified in [13, 14]. $H_{m^{2}}(\zeta)$-actions on path algebras of quivers were studied in [17].

In Section 3 we construct semisimple $H_{m^{2}}(\zeta)$-simple Lie algebras $L_{\alpha}(B)$ where $B$ is a finite dimensional simple Lie algebra and $\alpha \in F$ where $F$ is the base field. In Theorems 3.1 and 4.11 we prove that if $F$ is algebraically closed of characteristic 0 , then each finite dimensional $H_{m^{2}}(\zeta)$-simple $H_{m^{2}}(\zeta)$-module Lie algebra semisimple in ordinary sense either has the zero $v$-action or is isomorphic as an $H_{m^{2}}(\zeta)$-module Lie algebra to one of the Lie algebras $L_{\alpha}(B)$. In Theorem 3.3 we show that $L_{\alpha_{1}}\left(B_{1}\right) \cong L_{\alpha_{2}}\left(B_{2}\right)$ if and only if $B_{1} \cong B_{2}$ and $\alpha_{2}=\zeta^{k} \alpha_{1}$ for some $k \in \mathbb{Z}$.

In order to exclude the case of simple $H_{m^{2}}(\zeta)$-simple Lie algebras, which is done in Theorem 4.11, and treat the case of non-semisimple $H_{m^{2}}(\zeta)$-simple Lie algebras, in Section 4 we introduce $H_{m^{2}}(\zeta)$-simple Lie algebras $L(B, \gamma)$ where $\gamma \in F$ and $B$ is a simple Lie algebra. In Theorem 4.3 we show that $L\left(B, \frac{1}{\alpha^{m}(1-\zeta)^{m}}\right) \cong L_{\alpha}(B)$ as $H_{m^{2}}(\zeta)$-module Lie algebras for $\alpha \neq 0$. In Theorem 5.1 we prove that every non-semisimple $H_{m^{2}}(\zeta)$-simple Lie algebra is

2010 Mathematics Subject Classification. Primary 17B40; Secondary 17B01, 17B05, 17B40, 17B70, 16T05, $20 \mathrm{C} 30$.

Key words and phrases. Polynomial identity, $H$-module algebra, Taft algebra, codimension, PI-exponent, Lie algebra.

Supported by Fonds Wetenschappelijk Onderzoek — Vlaanderen post doctoral fellowship (Belgium). 
isomorphic to one of the Lie algebras $L(B, 0)$. It turns out that the nilpotent radical of each $H_{m^{2}}(\zeta)$-simple Lie algebra coincides with its solvable radical.

Although the classification of Lie algebras is in some sense parallel to the associative case, the Lie case requires different techniques. Furthermore, anti-commutativity of the commutator in a Lie algebra is a strong restriction on the $H_{m^{2}}(\zeta)$-action and we get much less possible parameters to describe $H_{m^{2}}(\zeta)$-simple Lie algebras. In addition, every finite dimensional $H_{m^{2}}(\zeta)$-module Lie algebra simple in the ordinary sense, is just a $\mathbb{Z}_{m}$-graded Lie algebra with the zero skew-derivation (Theorem 4.11), which is in contrast to the associative case [14, Theorem 1].

In Section 7 we apply the results obtained to codimensions of polynomial $H$-identities.

The codimension sequence $c_{n}(A)$ of polynomial identities of an algebra $A$ is an important numerical invariant of $A$. It turns out that the asymptotic behaviour of $c_{n}(A)$ is tightly related to the structure of $A[7,28]$.

In 1980s, S. A. Amitsur conjectured that if an associative algebra $A$ over a field of characteristic 0 satisfies a nontrivial polynomial identity, then there exists a PI-exponent $\lim _{n \rightarrow \infty} \sqrt[n]{c_{n}(A)} \in \mathbb{Z}_{+}$. The original Amitsur conjecture was proved by A. Giambruno and M. V. Zaicev [8] in 1999. Its analog for finite dimensional Lie algebras was proved by M. V. Zaicev [28] in 2002.

In general, the analog of Amitsur's conjecture for infinite dimensional Lie algebras is wrong. First, the codimension growth can be overexponential [27]. Second, the exponent of the codimension growth can be non-integer [19, 20]. It is still unknown whether there exist Lie algebras $L$ with

$$
\varliminf_{n \rightarrow \infty} \sqrt[n]{c_{n}(L)} \neq \varlimsup_{n \rightarrow \infty} \sqrt[n]{c_{n}(L)}
$$

Algebras endowed with an additional structure, e.g. grading, action of a group $G$, a Lie algebra or a Hopf algebra $H$, find their applications in many areas of mathematics and physics. For algebras with an additional structure, it is natural to consider the corresponding polynomial identities, namely, graded, differential, $G$ - and $H$-identities.

Polynomial $H$-identities have proved to be an important tool in the study of graded polynomial identities in graded algebras. In fact, they play a crucial role in the proof of the existence of an integer graded PI-exponent for an arbitrary group graded finite dimensional Lie algebra [11, Theorem 1]. In [11, Theorem 7] the author proved that for every finite dimensional semisimple Hopf algebra $H$ and every finite dimensional $H$-module Lie algebra there exists integer $\operatorname{PIexp}^{H}(L):=\lim _{n \rightarrow \infty} \sqrt[n]{c_{n}^{H}(L)}$ where $c_{n}^{H}(L)$ is the codimension sequence of polynomial $H$-identities of $L$, i.e. the analog of Amitsur's conjecture holds for $c_{n}^{H}(L)$. Later the analog of Amitsur's conjecture was proved for some other classes of $H$-module Lie algebras where the solvable and nilpotent radicals were still $H$-invariant 12 .

We believe that the analog of Amitsur's conjecture for $H$-module Lie algebras is true in the following form which belongs to Yu. A. Bahturin:

Conjecture 1.1. Let $L$ be a finite dimensional $H$-module Lie algebra for a Hopf algebra $H$ over a field of characteristic 0 . Then there exists an integer $\operatorname{PIexp}^{H}(L):=\lim _{n \rightarrow \infty} \sqrt[n]{c_{n}^{H}(L)}$.

In Theorem 7.1 we show this in the case when $L$ is an $H_{m^{2}}(\zeta)$-simple $H_{m^{2}}(\zeta)$-module Lie algebra over an algebraically closed field of characteristic 0 . In this case we have $\lim _{n \rightarrow \infty} \sqrt[n]{c_{n}^{H_{m^{2}}(\zeta)}(L)}=\operatorname{dim} L$.

\section{H-MODUle AlgEBRAS}

A (not necessarily associative) algebra $A$ over a field $F$ is a (left) $H$-module algebra for some Hopf algebra $H$ if $L$ is a (left) $H$-module such that $h(a b)=\left(h_{(1)} a\right)\left(h_{(2)} b\right)$ for all $h \in H$, 
$a, b \in A$. Here we use Sweedler's notation $\Delta h=h_{(1)} \otimes h_{(2)}$ where $\Delta$ is the comultiplication in $H$. We refer the reader to [3, 21, 25] for an account of Hopf algebras and algebras with Hopf algebra actions.

In the current article we study $H$-module Lie algebras $L$. The product of two elements $a, b \in L$ is denoted by $[a, b]$. We say that $L$ is $H$-simple if $[L, L] \neq 0$ and $L$ has no non-trivial $H$-invariant ideals.

Example 2.1. If $G$ is a group and $F$ is a field, then the group algebra $F G$ is a Hopf algebra where $\Delta(g)=g \otimes g, \varepsilon(g)=1$, and $S(g)=g^{-1}$ for all $g \in G$. If $G$ is acting on a Lie algebra $L$ by automorphisms, then the $G$-action can be extended by linearity to an $F G$-action such that $L$ is an $F G$-module Lie algebra.

Example 2.2. If $\mathfrak{g}$ is a Lie algebra over a field $F$, then its universal enveloping algebra $U(\mathfrak{g})$ is a Hopf algebra where $\Delta(a)=1 \otimes a+a \otimes 1, S(a)=-a, \varepsilon(a)=0$ for all $a \in \mathfrak{g}$. (The maps $\Delta: U(\mathfrak{g}) \rightarrow U(\mathfrak{g}) \otimes U(\mathfrak{g})$ and $\varepsilon: U(\mathfrak{g}) \rightarrow F$ are extended from $\mathfrak{g}$ as homomorphisms of algebras with 1 and the map $S: U(\mathfrak{g}) \rightarrow U(\mathfrak{g})$ is extended as an anti-homomorphism of algebras with 1.) If $\mathfrak{g}$ is acting on a Lie algebra $L$ by derivations, then the $\mathfrak{g}$-action can be naturally extended to a $U(\mathfrak{g})$-action such that $L$ is a $U(\mathfrak{g})$-module Lie algebra.

Let $G$ be a group. A Lie algebra $L=\bigoplus_{g \in G} L^{(g)}$ (direct sum of subspaces) is G-graded if $\left[L^{(g)}, L^{(h)}\right] \subseteq L^{(g h)}$ for all $g, h \in T$. A subspace $V$ of $L$ is graded (or homogeneous) if $V=\bigoplus_{g \in G} V \cap L^{(g)}$.

Consider the vector space $(F G)^{*}$ dual to $F G$. Then $(F G)^{*}$ is an associative algebra with the multiplication defined by $(h w)(g)=h(g) w(g)$ for $h, w \in(F G)^{*}$ and $g \in G$. The identity element is defined by $1_{(F G)^{*}}(g)=1$ for all $g \in G$. In other words, $(F G)^{*}$ is the algebra dual to the coalgebra $F G$.

If $L=\bigoplus_{g \in G} L^{(g)}$ is a $G$-graded Lie algebra, then we have the following natural $(F G)^{*}$ action on $L: h a^{(g)}:=h(g) a^{(g)}$ for all $h \in(F G)^{*}, a^{(g)} \in L^{(g)}$ and $g \in G$. If $G$ is a finite group, then $(F G)^{*}$ has a structure of a Hopf algebra and $L$ becomes an $(F G)^{*}$-module Lie algebra.

Let $m \geqslant 2$ be an integer and let $\zeta$ be a primitive $m$ th root of unity in a field $F$. (Such root exists in $F$ only if char $F \nmid m$.) Consider the associative algebra $H_{m^{2}}(\zeta)$ with unity generated by elements $c$ and $v$ satisfying the relations $c^{m}=1, v^{m}=0, v c=\zeta c v$. Note that $\left(c^{i} v^{k}\right)_{0 \leqslant i, k \leqslant m-1}$ is a basis of $H_{m^{2}}(\zeta)$. We introduce on $H_{m^{2}}(\zeta)$ a structure of a coalgebra by $\Delta(c)=c \otimes c, \Delta(v)=c \otimes v+v \otimes 1, \varepsilon(c)=1, \varepsilon(v)=0$. Then $H_{m^{2}}(\zeta)$ is a Hopf algebra with the antipode $S$ where $S(c)=c^{-1}$ and $S(v)=-c^{-1} v$. The algebra $H_{m^{2}}(\zeta)$ is called a Taft algebra.

In the paper, each time we consider $H_{m^{2}}(\zeta)$-module algebras, we implicitly assume that the base field $F$ contains a primitive $m$ th root of unity $\zeta$ and char $F \nmid m$.

Remark 2.3. Note that if $L$ is an $H_{m^{2}}(\zeta)$-module Lie algebra, then the cyclic group $\langle c\rangle_{m} \cong$ $\mathbb{Z}_{m}:=\mathbb{Z} / m \mathbb{Z}$ is acting on $L$ by automorphisms and $v$ is acting by a nilpotent skew-derivation. Every Lie algebra $L$ with a $\mathbb{Z}_{m}$-action by automorphisms is a $\mathbb{Z}_{m}$-graded Lie algebra:

$$
L=\bigoplus_{i=0}^{m-1} L^{(i)}, \quad L^{(i)}:=\left\{a \in L \mid c a=\zeta^{i} a\right\}, \quad L^{(i)} L^{(k)} \subseteq L^{(i+k)} .
$$

(When we consider $\mathbb{Z}_{m}$-gradings, all upper indices in parentheses are assumed to be modulo m.) Conversely, if $L=\bigoplus_{i=0}^{m-1} L^{(i)}$ is a $\mathbb{Z}_{m}$-graded Lie algebra, then $\mathbb{Z}_{m}$ is acting on $L$ by automorphisms: $c a^{(i)}:=\zeta^{i} a^{(i)}$ for all $a^{(i)} \in L^{(i)}$. Moreover, $L$ is $\mathbb{Z}_{m}$-simple (i.e. $[L, L] \neq 0$ and $L$ has no non-trivial $\mathbb{Z}_{m}$-invariant ideals) if and only if $L$ is $\mathbb{Z}_{m}$-graded simple (i.e. $[L, L] \neq 0$ and $L$ has no non-trivial ideals homogeneous in the $\mathbb{Z}_{m}$-grading). If $L$ is a $\mathbb{Z}_{m^{-}}$ graded Lie algebra, then its solvable and nilpotent radicals are $\mathbb{Z}_{m}$-graded ideals since they are stable under the automorphism $c$. 


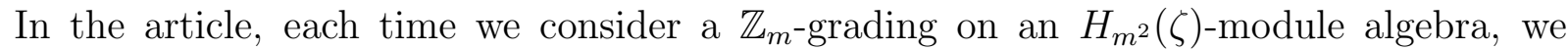
assume that this $\mathbb{Z}_{m}$-grading is induced by the $c$-action.

$\mathbb{Z}_{m}$-graded modules over $\mathbb{Z}_{m}$-graded Lie algebras are defined in the natural way. The analogs of the Weyl theorem on complete reducibility and the Jordan-Hölder Theorem hold for them. (The proof of the first one can be found e.g. in [9, Lemma 3] or [10, Theorem 9]. The second one is proved in the usual way considering graded submodules only.)

In the theorems below we use quantum binomial coefficients:

$$
\left(\begin{array}{l}
n \\
0
\end{array}\right)_{\zeta}=\left(\begin{array}{l}
n \\
n
\end{array}\right)_{\zeta}:=1, \quad\left(\begin{array}{l}
n \\
k
\end{array}\right)_{\zeta}:=\frac{n !_{\zeta}}{(n-k) !_{\zeta} k !_{\zeta}}
$$

where $n !_{\zeta}:=n_{\zeta}(n-1)_{\zeta} \cdots 1_{\zeta}$ and $n_{\zeta}:=1+\zeta+\zeta^{2}+\cdots+\zeta^{n-1}, n \in \mathbb{N}, 0_{\zeta}:=1$.

\section{Semisimple $H_{m^{2}}(\zeta)$-Simple Lie Algebras}

In this section we classify semisimple $H_{m^{2}}(\zeta)$-simple Lie algebras which are non-simple in the ordinary sense.

Let $B$ be a simple Lie algebra over a field $F$. Suppose $F$ contains a primitive $m$ th root of unity $\zeta$. Let $\alpha \in F$. Denote

$$
L_{\alpha}(B):=\underbrace{B \oplus \ldots \oplus B}_{m} \text { (direct sum of ideals). }
$$

Define the $c$ - and $v$-action on $L_{\alpha}(B)$ by

$$
c\left(a_{1}, a_{2}, \ldots, a_{m-1}, a_{m}\right)=\left(a_{m}, a_{1}, a_{2}, \ldots, a_{m-1}\right)
$$

and

$$
v\left(a_{1}, \ldots, a_{m}\right)=\alpha\left(a_{1}-a_{m}, \zeta\left(a_{2}-a_{1}\right), \ldots, \zeta^{m-1}\left(a_{m}-a_{m-1}\right)\right)
$$

for all $a_{1}, \ldots, a_{m} \in B$.

By induction (the details can be found in [14, Lemma 3]), for arbitrary $a_{1}, \ldots, a_{m} \in B$, we get

$$
v^{\ell}\left(a_{1}, a_{2}, \ldots, a_{m}\right)=\left(b_{1}, b_{2}, \ldots, b_{m}\right)
$$

where

$$
b_{k}=\alpha^{\ell} \zeta^{\ell(k-1)} \sum_{j=0}^{\ell}(-1)^{j} \zeta^{-\frac{j(j-1)}{2}}\left(\begin{array}{l}
\ell \\
j
\end{array}\right)_{\zeta^{-1}} a_{k-j}
$$

and $a_{-j}:=a_{m-j}$ for $j \geqslant 0$. Hence $v^{m}\left(a_{1}, a_{2}, \ldots, a_{m}\right)=0$ for all $a_{i} \in B$. An explicit check shows that (3.1) and (3.2) define the $H_{m^{2}}(\zeta)$-action on $L_{\alpha}(B)$ correctly and $L_{\alpha}(B)$ is $\mathbb{Z}_{m}$-graded simple and, therefore, $H_{m^{2}}(\zeta)$-simple.

Another description of $L_{\alpha}(B)$ for $\alpha \neq 0$ will be given in Theorem 4.3 below.

Theorem 3.1. Let $L$ be a finite dimensional semisimple $H_{m^{2}}(\zeta)$-simple Lie algebra over an algebraically closed field $F$ of characteristic 0 . Suppose $L$ is semisimple but not simple. Then $L$ is a $\mathbb{Z}_{m}$-graded simple Lie algebra. If $v L \neq 0$, then $L \cong L_{\alpha}(B)$ for some simple Lie algebra $B$ and some $\alpha \in F$.

Proof. First, $L=B_{1} \oplus \ldots \oplus B_{s}$ (direct sum of ideals) where $B_{i}$ are simple Lie algebras. Thus for every $1 \leqslant i \leqslant s$ there exists $1 \leqslant j(i) \leqslant s$ such that $c B_{i}=B_{j(i)}$. Moreover, $v[a, b]=$ $[c a, v b]+[v a, b] \in B_{i} \oplus B_{j(i)}$ for all $a, b \in B_{i}$. Since $\left[B_{i}, B_{i}\right]=B_{i}$, we get $v B_{i} \subseteq B_{i} \oplus B_{j(i)}$. In particular, the ideal $\sum_{k=0}^{m-1} c^{k} B_{1}$ is invariant under both $c$ and $v$. Since $L$ is $H_{m^{2}}(\zeta)$-simple, we get $\sum_{k=0}^{m-1} c^{k} B_{1}=L$ and, obviously, $L$ is $\mathbb{Z}_{m}$-simple and $\mathbb{Z}_{m}$-graded simple. Without loss of generality, we may assume that $B_{i}=c^{i-1} B_{1}$. 
Let $\pi_{i}: B \rightarrow B_{i}$ be the natural projection. Define $\rho_{i}: B_{i} \rightarrow B_{i}$ and $\theta_{i}: B_{i} \rightarrow c B_{i}$ by $\rho_{i}(a)=\pi_{i}(v a)$ and $\theta_{i}(a)=\pi_{i+1}(v a)$ for all $a \in B_{i}$. (In the proof of the theorem all lower indices are assumed to be modulo $s$, e.g. $\pi_{s+1}:=\pi_{1}$.) Then

$$
\rho_{i}[a, b]=\pi_{i}(v[a, b])=\pi_{i}([c a, v b]+[v a, b])=\left[\rho_{i}(a), b\right] .
$$

Analogously,

$$
\theta_{i}[a, b]=\pi_{i+1}(v[a, b])=\pi_{i+1}([c a, v b]+[v a, b])=\left[c a, \theta_{i}(b)\right]
$$

for all $a, b \in B_{i}$. In particular, both $\rho_{i}$ and $\theta_{i}$ are homomorphisms of $B_{i}$-modules.

Since $B_{i}$ are simple Lie algebras, $B_{i}$ are irreducible $B_{i}$-modules. Hence by the Schur lemma we have $\rho_{i}=\alpha_{i} \operatorname{id}_{B_{i}}$ and $\theta_{i}=\beta_{i}\left(\left.c\right|_{B_{i}}\right)$ for some $\alpha_{i}, \beta_{i} \in F$. Since $v c=\zeta c v$, we have

$$
\alpha_{i+1} c a=\rho_{i+1}(c a)=\zeta \pi_{i+1}(c(v(a)))=\zeta c\left(\rho_{i}(a)\right)=\zeta \alpha_{i} c a
$$

and

$$
\beta_{i+1} c^{2} a=\theta_{i+1}(c a)=\zeta \pi_{i+2}(c(v(a)))=\zeta c\left(\theta_{i}(a)\right)=\zeta \beta_{i} c^{2} a
$$

for all $1 \leqslant i \leqslant s$ and $a \in B_{i}$. Hence $\alpha_{i}=\zeta^{i-1} \alpha_{1}$ and $\beta_{i}=\zeta^{i-1} \beta_{1}$ for all $1 \leqslant i \leqslant s$. Moreover, if at least one of $\alpha_{1}$ and $\beta_{1}$ is nonzero, we get $\zeta^{s}=1$ and $s=m$.

Note that for all $1 \leqslant i \leqslant s, a \in B_{i}$, and $b \in B_{i+1}$ we have

$$
0=v[a, b]=[c a, v b]+[v a, b]=\left[c a, \rho_{i+1}(b)\right]+\left[\theta_{i}(a), b\right]=\left(\alpha_{i+1}+\beta_{i}\right)[c a, b] .
$$

Since $\left[B_{i+1}, B_{i+1}\right]=B_{i+1}$, we obtain $\beta_{i}=-\alpha_{i+1}$ for all $1 \leqslant i \leqslant s$.

If $\alpha_{1}=0$, then $v L=0$ and the theorem is proved. Suppose $\alpha_{1} \neq 0$. Then $s=m$. Since $B_{i}=c^{i-1} B_{1}$ and $v a=\rho_{i}(a)+\theta_{i}(a)$ for all $a \in B_{i}$, we may identify $B_{i}$ and assume that $L=\underbrace{B \oplus \ldots \oplus B}_{m}$ (direct sum of ideals) for the simple Lie algebra $B:=B_{1}$, where 3.1) and (3.2) hold for $\alpha:=\alpha_{1}$.

Remark 3.2. If $v L=0$, then the proof of Theorem 3.1 shows that there exists $s \in \mathbb{N}, s \mid m$, and a simple Lie algebra $B$ with an action of the cyclic group of order $\frac{m}{s}$ with a generator $d$ such that

$$
\begin{gathered}
L \cong \underbrace{B \oplus \ldots \oplus B}_{s} \text { (direct sum of ideals) } \\
c\left(a_{1}, a_{2}, \ldots, a_{m-1}, a_{m}\right)=\left(d a_{m}, a_{1}, a_{2}, \ldots, a_{m-1}\right)
\end{gathered}
$$

and

$$
v\left(a_{1}, \ldots, a_{m}\right)=0
$$

for all $a_{1}, \ldots, a_{m} \in B$.

In Theorem 3.3 below we give necessary and sufficient conditions for $L_{\alpha_{1}}\left(B_{1}\right) \cong L_{\alpha_{2}}\left(B_{2}\right)$ as $H_{m^{2}}(\zeta)$-module Lie algebras.

Theorem 3.3. Let $B_{1}, B_{2}$ be simple Lie algebras over a field $F, \alpha_{1}, \alpha_{2} \in F$. Let $\zeta$ be a primitive mth root of unity. Suppose $\theta: L_{\alpha_{1}}\left(B_{1}\right) \widetilde{\Im} L_{\alpha_{2}}\left(B_{2}\right)$ is an isomorphism of Lie algebras and $H_{m^{2}}(\zeta)$-modules. Then there exists $0 \leqslant k \leqslant m-1$ and an isomorphism of Lie algebras $\psi: B_{1} \stackrel{\rightarrow}{\rightarrow} B_{2}$ such that

$$
\theta\left(b_{1}, \ldots, b_{m}\right)=\left(\psi\left(b_{k+1}\right), \ldots, \psi\left(b_{m}\right), \psi\left(b_{1}\right), \ldots, \psi\left(b_{k}\right)\right)
$$

for all $b_{i} \in B_{1}$. Moreover, $\alpha_{2}=\zeta^{k} \alpha_{1}$. Conversely, if $B_{1} \cong B_{2}$ as ordinary Lie algebras and $\alpha_{2}=\zeta^{k} \alpha_{1}$ for some $k \in \mathbb{Z}$, then $L_{\alpha_{1}}\left(B_{1}\right) \cong L_{\alpha_{2}}\left(B_{2}\right)$ as $H_{m^{2}}(\zeta)$-module Lie algebras. 
Proof. Note that each minimal ideal of $L_{\alpha_{2}}\left(B_{2}\right)$ coincides with one of the copies of $B_{2}$. Thus there exists $0 \leqslant k \leqslant m-1$ such that

$$
\theta\left(B_{1}, 0, \ldots, 0\right)=(\underbrace{0, \ldots, 0}_{m-k}, B_{2}, 0, \ldots, 0) .
$$

Denote the induced isomorphism $B_{1} \widetilde{\rightarrow} B_{2}$ by $\psi$. Then

$$
\theta(b, 0, \ldots, 0)=(\underbrace{0, \ldots, 0}_{m-k}, \psi(b), 0, \ldots, 0)
$$

for all $b \in B$. Now (3.1) together with $\theta(c a)=c \theta(a)$ for all $a \in L_{\alpha_{1}}\left(B_{1}\right)$ implies 3.3). Using (3.2) and $\theta(v a)=v \theta(a)$ for all $a \in L_{\alpha_{1}}\left(B_{1}\right)$, we get $\alpha_{2}=\zeta^{k} \alpha_{1}$. The converse is now evident.

Remark 3.4. In particular, if $\alpha \neq 0$, then all automorphisms of $L_{\alpha}(B)$ as an $H_{m^{2}}(\zeta)$-module Lie algebra are induced by automorphisms of $B$ as an ordinary Lie algebra, and the corresponding automorphisms groups $\operatorname{Aut}_{H_{m^{2}}(\zeta)}\left(L_{\alpha}(B)\right)$ and $\operatorname{Aut}(B)$ can be identified. If $\alpha=0$, then $\operatorname{Aut}_{H_{m^{2}}(\zeta)}\left(L_{\alpha}(B)\right) \cong \operatorname{Aut}(B) \times \mathbb{Z}_{m}$.

\section{Lie Algebras $L(B, \gamma)$ And $H_{m^{2}}(\zeta)$-ACtions on simple Lie Algebras}

The next step in the classification of finite dimensional $H_{m^{2}}(\zeta)$-simple Lie algebras is the study of $H_{m^{2}}(\zeta)$-actions on simple Lie algebras. In fact, we will prove that finite dimensional simple Lie algebras $L$ endowed with $H_{m^{2}}(\zeta)$-action have $v a=0$ for all $a \in L$. (See Theorem 4.11 below.) In order to do this, we introduce $H_{m^{2}}(\zeta)$-simple Lie algebras $L(B, \gamma)$.

Theorem 4.1. Let $B$ be a simple Lie algebra over a field $F$ and let $\gamma \in F$ be some element. Suppose $F$ contains some primitive $m$ th root of unity $\zeta$. Define vector spaces $L^{(i)}, 1 \leqslant i \leqslant$ $m-1, F$-linearly isomorphic to $L^{(0)}:=B$. Let $\varphi: L^{(i-1)} \underset{\rightarrow}{\rightarrow} L^{(i)}, 1 \leqslant i \leqslant m-1$, be the corresponding F-linear bijections, which we denote by the same letter. Let $\varphi\left(L^{(m-1)}\right):=0$. Consider the $H_{m^{2}}(\zeta)$-module $L(B, \gamma):=\bigoplus_{i=0}^{m-1} L^{(i)}$ (direct sum of subspaces) where $v \varphi(a)=$ a for all $a \in L^{(i)}, 0 \leqslant i \leqslant m-2, v B=0$, and $c a^{(i)}=\zeta^{i} a^{(i)}$, $a^{(i)} \in L^{(i)}$. Define the commutator on $L(B, \gamma)$ by

$$
\left[\varphi^{k}(a), \varphi^{\ell}(b)\right]:=\left\{\begin{aligned}
\left(\begin{array}{c}
k+\ell \\
k
\end{array}\right)_{\zeta} \varphi^{k+\ell}[a, b] \quad \text { if } \quad k+\ell<m, \\
\gamma \frac{(k+\ell-m) !_{\zeta}}{k !_{\zeta} \ell !_{\zeta}} \varphi^{k+\ell-m}[a, b] \quad \text { if } \quad k+\ell \geqslant m
\end{aligned}\right.
$$

for all $a, b \in B$ and $0 \leqslant k, \ell<m$. Then $L(B, \gamma)$ is an $H_{m^{2}}(\zeta)$-simple Lie algebra.

Proof. An explicit verification shows that the formulas indeed define on $L(B, \gamma)$ a structure of an $H_{m^{2}}(\zeta)$-module Lie algebra. Here we check only that $v[u, w]=\left[v_{(1)} u, v_{(2)} w\right]$ for all $u, w \in L(B, \gamma)$.

Let $0 \leqslant k, \ell<m$ and $a, b \in B$. If $k=\ell=0$, then $v[a, b]=0=[c a, v b]+[v a, b]$.

If $k=0, \ell>0$, then

$$
v\left[a, \varphi^{\ell}(b)\right]=v \varphi^{\ell}[a, b]=\varphi^{\ell-1}[a, b]=\left[a, \varphi^{\ell-1}(b)\right]=\left[c a, v \varphi^{\ell}(b)\right]+\left[v a, \varphi^{\ell}(b)\right] .
$$

If $k>0, \ell=0$, then

$$
v\left[\varphi^{k}(a), b\right]=v \varphi^{k}[a, b]=\varphi^{k-1}[a, b]=\left[\varphi^{k-1}(a), b\right]=\left[c \varphi^{k}(a), v b\right]+\left[v \varphi^{k}(a), b\right] .
$$

If $k, \ell>0, k+\ell<m$, then

$$
\begin{aligned}
v\left[\varphi^{k}(a), \varphi^{\ell}(b)\right]= & \left(\begin{array}{c}
k+\ell \\
k
\end{array}\right)_{\zeta} \varphi^{k+\ell-1}[a, b]=\left(\zeta^{k}\left(\begin{array}{c}
k+\ell-1 \\
k
\end{array}\right)_{\zeta}+\left(\begin{array}{c}
k+\ell-1 \\
k-1
\end{array}\right)_{\zeta}\right) \varphi^{k+\ell-1}[a, b] \\
& =\left[c \varphi^{k}(a), \varphi^{\ell-1}(b)\right]+\left[\varphi^{k-1}(a), \varphi^{\ell}(b)\right]=\left[c \varphi^{k}(a), v \varphi^{\ell}(b)\right]+\left[v \varphi^{k}(a), \varphi^{\ell}(b)\right]
\end{aligned}
$$


since

$$
\begin{array}{r}
\zeta^{k}\left(\begin{array}{c}
k+\ell-1 \\
k
\end{array}\right)_{\zeta}+\left(\begin{array}{c}
k+\ell-1 \\
k-1
\end{array}\right)_{\zeta}=\frac{\zeta^{k}(k+\ell-1) !_{\zeta}}{k !_{\zeta}(\ell-1) !_{\zeta}}+\frac{(k+\ell-1) !_{\zeta}}{(k-1) !_{\zeta} \ell !_{\zeta}} \\
=\left(\zeta^{k} \ell_{\zeta}+k_{\zeta}\right) \frac{(k+\ell-1) !_{\zeta}}{k !_{\zeta} \ell !_{\zeta}}=(k+\ell)_{\zeta} \frac{(k+\ell-1) !_{\zeta}}{k !_{\zeta} \ell !_{\zeta}}=\frac{(k+\ell) !_{\zeta}}{k !_{\zeta} \ell !_{\zeta}}=\left(\begin{array}{c}
k+\ell \\
k
\end{array}\right)_{\zeta} .
\end{array}
$$

If $k+\ell \geqslant m$, then $k, \ell>0$. If $k+\ell=m$, then $(k+\ell)_{\zeta}=m_{\zeta}=0$ and

$$
\begin{array}{r}
v\left[\varphi^{k}(a), \varphi^{\ell}(b)\right]=\frac{\gamma}{k !_{\zeta} \ell !_{\zeta}} v[a, b]=0=\left(\begin{array}{c}
k+\ell \\
k
\end{array}\right)_{\zeta} \varphi^{k+\ell-1}[a, b] \\
=\left(\zeta^{k}\left(\begin{array}{c}
k+\ell-1 \\
k
\end{array}\right)_{\zeta}+\left(\begin{array}{c}
k+\ell-1 \\
k-1
\end{array}\right)_{\zeta}\right) \varphi^{k+\ell-1}[a, b]=\left[c \varphi^{k}(a), \varphi^{\ell-1}(b)\right]+\left[\varphi^{k-1}(a), \varphi^{\ell}(b)\right] \\
=\left[c \varphi^{k}(a), v \varphi^{\ell}(b)\right]+\left[v \varphi^{k}(a), \varphi^{\ell}(b)\right] .
\end{array}
$$

If $k+\ell>m$, then $(k+\ell)_{\zeta}=(k+\ell-m)_{\zeta}+\zeta^{k+\ell-m} m_{\zeta}=(k+\ell-m)_{\zeta}$ and

$$
\begin{array}{r}
v\left[\varphi^{k}(a), \varphi^{\ell}(b)\right]=\gamma \frac{(k+\ell-m) !_{\zeta}}{k !_{\zeta} \ell !_{\zeta}} \varphi^{k+\ell-m-1}[a, b] \\
=\gamma(k+\ell)_{\zeta} \frac{(k+\ell-m-1) !_{\zeta}}{k !_{\zeta} \ell !_{\zeta}} \varphi^{k+\ell-m-1}[a, b] \\
=\gamma\left(\zeta^{k} \frac{(k+\ell-m-1) !_{\zeta}}{k !_{\zeta}(\ell-1) !_{\zeta}}+\frac{(k+\ell-m-1) !_{\zeta}}{(k-1) !_{\zeta} \ell !_{\zeta}}\right) \varphi^{k+\ell-m-1}[a, b] \\
=\left[c \varphi^{k}(a), \varphi^{\ell-1}(b)\right]+\left[\varphi^{k-1}(a), \varphi^{\ell}(b)\right] \\
=\left[c \varphi^{k}(a), v \varphi^{\ell}(b)\right]+\left[v \varphi^{k}(a), \varphi^{\ell}(b)\right] .
\end{array}
$$

We have considered all possible variants for $0 \leqslant k, \ell<m$. Hence $v[u, w]=\left[v_{(1)} u, v_{(2)} w\right]$ for all $u, w \in L(B, \gamma)$.

Suppose that $I$ is an $H_{m^{2}}(\zeta)$-invariant ideal of $L$. Then $v^{m} I=0$. Let $t \in \mathbb{Z}_{+}$such that $v^{t} I \neq 0, v^{t+1} I=0$. Then $0 \neq v^{t} I \subseteq I \cap \operatorname{ker} v$. However, ker $v=B$ is a simple Lie algebra. Thus $I \cap \operatorname{ker} v=\operatorname{ker} v$ and ker $v \subseteq I$. Since

$$
\left[\operatorname{ker} v, L^{(i)}\right]=\left[B, \varphi^{i}(B)\right]=\varphi^{i}[B, B]=\varphi^{i}(B)=L^{(i)} \text { for all } 1 \leqslant i \leqslant m-1,
$$

we get $I=L(B, \gamma)$. Therefore, $L(B, \gamma)$ is an $H_{m^{2}}(\zeta)$-simple Lie algebra.

Remark 4.2. Lie algebras $L(B, 0)$ are not semisimple. The solvable radical of $L(B, 0)$ coincides with the nilpotent radical and equals $\bigoplus_{i=1}^{m-1} L^{(i)}$.

In Theorem 4.3 below we prove that if the field $F$ is algebraically closed and $\gamma \neq 0$, then $L(B, \gamma)$ is isomorphic to one of the non-simple $\mathbb{Z}_{m}$-graded simple $H_{m^{2}}(\zeta)$-module Lie algebras $L_{\alpha}(B)$ defined in Section 3 .

Theorem 4.3. Let $B$ be a simple Lie algebra over a field $F$. Suppose $F$ contains some primitive mth root of unity $\zeta$. Let $\alpha \in F, \alpha \neq 0$. Then $L\left(B, \frac{1}{\alpha^{m}(1-\zeta)^{m}}\right) \cong L_{\alpha}(B)$ as $H_{m^{2}}(\zeta)$ module Lie algebras.

Proof. Note that

$$
L_{\alpha}(B)^{(k)}=\left\{\left(b, \zeta^{-k} b, \zeta^{-2 k} b, \ldots, \zeta^{-(m-1) k} b\right) \mid b \in B\right\}
$$

for $0 \leqslant k \leqslant m-1$. In particular, $L_{\alpha}(B)^{(0)} \cong B$. Define

$$
\varphi\left(b, \zeta^{-k} b, \zeta^{-2 k} b, \ldots, \zeta^{-(m-1) k} b\right):=\frac{1}{\alpha\left(1-\zeta^{k+1}\right)}\left(b, \zeta^{-(k+1)} b, \zeta^{-2(k+1)} b, \ldots, \zeta^{-(m-1)(k+1)} b\right)
$$


for all $b \in B$ and $0 \leqslant k<m-1$. Then

$$
\begin{array}{r}
\varphi^{k}(b, b, \ldots, b)=\frac{1}{\alpha^{k}(1-\zeta)\left(1-\zeta^{2}\right) \ldots\left(1-\zeta^{k}\right)}\left(b, \zeta^{-k} b, \zeta^{-2 k} b, \ldots, \zeta^{-(m-1) k} b\right) \\
=\frac{1}{\alpha^{k}(1-\zeta)^{k} k !_{\zeta}}\left(b, \zeta^{-k} b, \zeta^{-2 k} b, \ldots, \zeta^{-(m-1) k} b\right) .
\end{array}
$$

Note that $L_{\alpha}(B)^{(k)}=\varphi^{k}\left(L_{\alpha}(B)^{(0)}\right)$ and $v \varphi(a)=a$ for all $a \in L_{\alpha}(B)^{(k)}, 0 \leqslant k<m-1$. Moreover, $\left[\varphi^{k}(a), \varphi^{\ell}(b)\right]$ can be calculated using 4.1 for $\gamma=\frac{1}{\alpha^{m}(1-\zeta)^{m}}$ for all $a, b \in L_{\alpha}(B)^{(0)}$ and $0 \leqslant k, \ell<m$. Hence $L_{\alpha}(B) \cong L\left(B, \frac{1}{\alpha^{m}(1-\zeta)^{m}}\right)$ as $H_{m^{2}}(\zeta)$-module Lie algebras.

Now we prove several lemmas on $H_{m^{2}}(\zeta)$-module Lie algebras.

Lemma 4.4. Let $L$ be an $H_{m^{2}}(\zeta)$-module Lie algebra over a field $F$. Then

$$
\begin{gathered}
\left(\zeta^{k}-1\right)\left[a^{(k)}, v b^{(\ell)}\right]=\left(\zeta^{\ell}-1\right)\left[v a^{(k)}, b^{(\ell)}\right], \\
\left(\zeta^{\ell}-1\right) v\left[a^{(k)}, b^{(\ell)}\right]=\left(\zeta^{k+\ell}-1\right)\left[a^{(k)}, v b^{(\ell)}\right]
\end{gathered}
$$

for all $a^{(k)} \in L^{(k)}, b^{(\ell)} \in L^{(\ell)}$ in the natural $\mathbb{Z}_{m}$-grading induced by the c-action. Moreover, if $L$ is a $\mathbb{Z}_{m}$-graded simple Lie algebra with respect to this grading, $v L^{(0)}=0$.

Proof. Note that

$$
v\left[a^{(k)}, b^{(\ell)}\right]=\left[c a^{(k)}, v b^{(\ell)}\right]+\left[v a^{(k)}, b^{(\ell)}\right]=\zeta^{k}\left[a^{(k)}, v b^{(\ell)}\right]+\left[v a^{(k)}, b^{(\ell)}\right]
$$

for all $a^{(k)} \in L^{(k)}$ and $b^{(\ell)} \in L^{(\ell)}$. At the same time

$$
\begin{aligned}
v\left[a^{(k)}, b^{(\ell)}\right]=-v\left[b^{(\ell)}, a^{(k)}\right] & =-\left[c b^{(\ell)}, v a^{(k)}\right]-\left[v b^{(\ell)}, a^{(k)}\right] \\
=-\zeta^{\ell}\left[b^{(\ell)}, v a^{(k)}\right]-\left[v b^{(\ell)}, a^{(k)}\right] & =\left[a^{(k)}, v b^{(\ell)}\right]+\zeta^{\ell}\left[v a^{(k)}, b^{(\ell)}\right] .
\end{aligned}
$$

Hence we obtain (4.3) and (4.4) and if $\ell \neq 0$, we get

$$
v\left[a^{(k)}, b^{(\ell)}\right]=\frac{\zeta^{k+\ell}-1}{\zeta^{\ell}-1}\left[a^{(k)}, v b^{(\ell)}\right] .
$$

In particular, $v\left[L^{(k)}, L^{(m-k)}\right]=0$ for all $1 \leqslant k \leqslant m-1$.

Suppose $L$ is a $\mathbb{Z}_{m}$-graded simple Lie algebra. If $L=L^{(0)}$, then $c$ is acting trivially and $v c=\zeta c v$ implies $v L=0$. Therefore, we may assume that $L \neq L^{(0)}$. Let $a \in L^{(k)}, k \neq 0, a \neq$ 0 . Since $L$ is $\mathbb{Z}_{m}$-graded simple and $a$ is homogeneous, $a$ generates $L$ as an ideal. Thus $L^{(0)}$ is an $F$-linear span of elements $\left[a, a_{1}, \ldots, a_{n}\right]$, where $a_{i} \in L^{\left(k_{i}\right)}, 0 \leqslant k_{i} \leqslant m-1, m \mid\left(k+k_{1}+\right.$ $\left.\ldots+k_{n}\right), n \in \mathbb{N}$. (Here we use long commutators $\left[x_{1}, \ldots, x_{n}\right]:=\left[\left[\ldots\left[\left[x_{1}, x_{2}\right], x_{3}\right], \ldots\right], x_{n}\right]$ ) If $k_{n} \neq 0$, then $\left[a, a_{1}, \ldots, a_{n}\right]=\left[\left[a, a_{1}, \ldots, a_{n-1}\right], a_{n}\right]$ implies $\left[a, a_{1}, \ldots, a_{n}\right] \in\left[L^{\left(m-k_{n}\right)}, L^{\left(k_{n}\right)}\right]$ and $v\left[a, a_{1}, \ldots, a_{n}\right]=0$. If $k_{n}=0$, we apply the Jacobi identity and rewrite $\left[a, a_{1}, \ldots, a_{n}\right]$ as a sum of $\left[\left[a, a_{n}\right], a_{1}, \ldots, a_{n-1}\right],\left[a,\left[a_{1}, a_{n}\right], \ldots, a_{n-1}\right]$, and $\left[a, a_{1}, \ldots,\left[a_{n-1}, a_{n}\right]\right]$. If $a_{n-1} \in L^{(0)}$, we continue this procedure. Finally, we get the situation where the last components in long commutators belong to $L^{\left(k_{i}\right)}, k_{i} \neq 0$. Applying the arguments used above, we get $v\left[a, a_{1}, \ldots, a_{n}\right]=0$. Hence $v L^{(0)}=0$.

Lemma 4.5. Let $L$ be an $H_{m^{2}}(\zeta)$-module Lie algebra over a field $F$ of characteristic char $F \nmid$ $m$, char $F \neq 2$. Let $a^{(\ell)} \in L^{(\ell)}, b^{(k)} \in L^{(k)}, u^{(m-k)} \in L^{(m-k)}$ for some $1 \leqslant k, \ell \leqslant m-1$. Then

$$
v\left[a^{(\ell)},\left[b^{(k)}, u^{(m-k)}\right]\right]=\frac{\zeta^{\ell}-1}{\zeta^{m-k}-1}\left[a^{(\ell)},\left[b^{(k)}, v u^{(m-k)}\right]\right] .
$$


Proof. By (4.3), (4.4) and the Jacobi identity,

$$
\begin{array}{r}
{\left[a^{(\ell)},\left[b^{(k)}, v u^{(m-k)}\right]\right]=-\frac{\zeta^{m-k}-1}{\zeta^{k}-1}\left[a^{(\ell)},\left[u^{(m-k)}, v b^{(k)}\right]\right]} \\
=-\frac{\zeta^{m-k}-1}{\zeta^{k}-1}\left(\left[\left[a^{(\ell)}, u^{(m-k)}\right], v b^{(k)}\right]+\left[u^{(m-k)},\left[a^{(\ell)}, v b^{(k)}\right]\right]\right) \\
=-\frac{\zeta^{m-k}-1}{\zeta^{\ell}-1} v\left[a^{(\ell)}, u^{(m-k)}, b^{(k)}\right]+\frac{\zeta^{m-k}-1}{\zeta^{\ell}-1}\left[u^{(m-k)},\left[b^{(k)}, v a^{(\ell)}\right]\right] \\
=-\frac{\zeta^{m-k}-1}{\zeta^{\ell}-1} v\left[a^{(\ell)}, u^{(m-k)}, b^{(k)}\right]+\frac{\zeta^{m-k}-1}{\zeta^{\ell}-1}\left(\left[\left[u^{(m-k)}, b^{(k)}\right], v a^{(\ell)}\right]+\left[b^{(k)},\left[u^{(m-k)}, v a^{(\ell)}\right]\right]\right) \\
=\frac{\zeta^{m-k}-1}{\zeta^{\ell}-1} v\left(-\left[a^{(\ell)}, u^{(m-k)}, b^{(k)}\right]+\left[u^{(m-k)}, b^{(k)}, a^{(\ell)}\right]\right)-\left[b^{(k)},\left[a^{(\ell)}, v u^{(m-k)}\right]\right] \\
=\frac{\zeta^{m-k}-1}{\zeta^{\ell}-1} v\left(-\left[a^{(\ell)}, u^{(m-k)}, b^{(k)}\right]+\left[u^{(m-k)}, b^{(k)}, a^{(\ell)}\right]\right) \\
-\left[\left[b^{(k)}, a^{(\ell)}\right], v u^{(m-k)}\right]-\left[a^{(\ell)},\left[b^{(k)}, v u^{(m-k)}\right]\right] \\
=\frac{\zeta^{m-k}-1}{\zeta^{\ell}-1} v\left(-\left[a^{(\ell)}, u^{(m-k)}, b^{(k)}\right]+\left[u^{(m-k)}, b^{(k)}, a^{(\ell)}\right]-\left[b^{(k)}, a^{(\ell)}, u^{(m-k)}\right]\right) \\
-\left[a^{(\ell)},\left[b^{(k)}, v u^{(m-k)}\right]\right] \\
=2 \frac{\zeta^{m-k}-1}{\zeta^{\ell}-1} v\left[a^{(\ell)},\left[b^{(k)}, u^{(m-k)}\right]\right]-\left[a^{(\ell)},\left[b^{(k)}, v u^{(m-k)}\right]\right]
\end{array}
$$

and the lemma follows.

Lemma 4.6. Let $L$ be an $H_{m^{2}}(\zeta)$-module Lie algebra over a field $F$ of characteristic char $F \nmid$ $m$, char $F \neq 2$. Suppose $L$ is a $\mathbb{Z}_{m}$-graded simple Lie algebra. Let $s \geqslant 2$ and $0 \leqslant k_{i} \leqslant m-1$ for $1 \leqslant i \leqslant s, k_{s}>0$. Let $a_{i}^{\left(k_{i}\right)} \in L_{i}^{\left(k_{i}\right)}$ for $1 \leqslant i \leqslant s$. Then

$$
v\left[a_{1}^{\left(k_{1}\right)},\left[a_{2}^{\left(k_{2}\right)}, \ldots,\left[a_{s-1}^{\left(k_{s-1}\right)}, a_{s}^{\left(k_{s}\right)}\right] \ldots\right]=\frac{\zeta^{\sum_{i=1}^{s} k_{i}}-1}{\zeta^{k_{s}}-1}\left[a_{1}^{\left(k_{1}\right)},\left[a_{2}^{\left(k_{2}\right)}, \ldots,\left[a_{s-1}^{\left(k_{s-1}\right)}, v a_{s}^{\left(k_{s}\right)}\right] \ldots\right] .\right.\right.
$$

Proof. We prove the assertion by induction on $s$. The base $s=2$ is a consequence of (4.4). Suppose $s>2$.

If $m \nmid \sum_{i=2}^{s} k_{i}$, then by (4.4) and the induction assumption,

$$
\begin{aligned}
v\left[a_{1}^{\left(k_{1}\right)},\left[a_{2}^{\left(k_{2}\right)}, \ldots,\left[a_{s-1}^{\left(k_{s-1}\right)}, a_{s}^{\left(k_{s}\right)}\right] \ldots\right]\right. & =\frac{\zeta^{\sum_{i=1}^{s} k_{i}}-1}{\zeta^{\sum_{i=2}^{s} k_{i}}-1}\left[a_{1}^{\left(k_{1}\right)}, v\left[a_{2}^{\left(k_{2}\right)}, \ldots,\left[a_{s-1}^{\left(k_{s-1}\right)}, a_{s}^{\left(k_{s}\right)}\right] \ldots\right]\right. \\
& =\frac{\zeta^{\sum_{i=1}^{s} k_{i}}-1}{\zeta^{k_{s}}-1}\left[a_{1}^{\left(k_{1}\right)},\left[a_{2}^{\left(k_{2}\right)}, \ldots,\left[a_{s-1}^{\left(k_{s-1}\right)}, v a_{s}^{\left(k_{s}\right)}\right] \ldots\right]\right.
\end{aligned}
$$

and the lemma follows.

Suppose $k_{i}=0$ for some $1 \leqslant i<s$. Then by the Jacobi identity (here the symbol $\widehat{a_{i}^{\left(k_{i}\right)}}$ means that the element $a_{i}^{\left(k_{i}\right)}$ is omitted),

$$
\begin{array}{r}
a:=v\left[a_{1}^{\left(k_{1}\right)},\left[a_{2}^{\left(k_{2}\right)}, \ldots,\left[a_{s-1}^{\left(k_{s-1}\right)}, a_{s}^{\left(k_{s}\right)}\right] \ldots\right]\right. \\
=\sum_{j=i+1}^{s-1} v\left[a_{1}^{\left(k_{1}\right)},\left[a_{2}^{\left(k_{2}\right)}, \ldots, \widehat{a_{i}^{\left(k_{i}\right)}}, \ldots,\left[a_{j-1}^{\left(k_{j-1}\right)},\left[\left[a_{i}^{\left(k_{i}\right)}, a_{j}^{\left(k_{j}\right)}\right],\left[a_{j+1}^{\left(k_{j+1}\right)}, \ldots\left[a_{s-1}^{\left(k_{s-1}\right)}, a_{s}^{\left(k_{s}\right)}\right] \ldots\right]\right.\right.\right.\right. \\
+v\left[a_{1}^{\left(k_{1}\right)},\left[a_{2}^{\left(k_{2}\right)}, \ldots,\left[\widehat{a_{i}^{\left(k_{i}\right)}}, \ldots,\left[a_{s-1}^{\left(k_{s-1}\right)},\left[a_{i}^{\left(k_{i}\right)}, a_{s}^{\left(k_{s}\right)}\right]\right] \ldots\right] .\right.\right.
\end{array}
$$


Since $k_{i}=0$, each $\left[a_{i}^{\left(k_{i}\right)}, a_{j}^{\left(k_{j}\right)}\right]$ is again of degree $k_{j}$ and we treat it as a single element. Applying the induction assumption for $s-1$, we get

$$
\begin{array}{r}
a=\frac{\zeta^{\sum_{i=1}^{s} k_{i}}-1}{\zeta^{k_{s}}-1} \\
\left(\sum _ { j = i + 1 } ^ { s - 1 } \left[a_{1}^{\left(k_{1}\right)},\left[a_{2}^{\left(k_{2}\right)}, \ldots, \widehat{a_{i}^{\left(k_{i}\right)}}, \ldots,\left[a_{j-1}^{\left(k_{j-1}\right)},\left[\left[a_{i}^{\left(k_{i}\right)}, a_{j}^{\left(k_{j}\right)}\right],\left[a_{j+1}^{\left(k_{j+1}\right)}, \ldots\left[a_{s-1}^{\left(k_{s-1}\right)}, v a_{s}^{\left(k_{s}\right)}\right] \ldots\right]\right.\right.\right.\right.\right. \\
+\left[a_{1}^{\left(k_{1}\right)},\left[a_{2}^{\left(k_{2}\right)}, \ldots,\left[\widehat{a_{i}^{\left(k_{i}\right)}}, \ldots,\left[a_{s-1}^{\left(k_{s-1}\right)}, v\left[a_{i}^{\left(k_{i}\right)}, a_{s}^{\left(k_{s}\right)}\right]\right] \ldots\right]\right) .\right.
\end{array}
$$

By (4.4) and the Jacobi identity, we get

$$
\begin{array}{r}
a=\frac{\zeta^{\sum_{i=1}^{s} k_{i}}-1}{\zeta^{k_{s}}-1} \\
\cdot\left(\sum _ { j = i + 1 } ^ { s - 1 } \left[a_{1}^{\left(k_{1}\right)},\left[a_{2}^{\left(k_{2}\right)}, \ldots, \widehat{a_{i}^{\left(k_{i}\right)}}, \ldots,\left[a_{j-1}^{\left(k_{j-1}\right)},\left[\left[a_{i}^{\left(k_{i}\right)}, a_{j}^{\left(k_{j}\right)}\right],\left[a_{j+1}^{\left(k_{j+1}\right)}, \ldots\left[a_{s-1}^{\left(k_{s-1}\right)}, v a_{s}^{\left(k_{s}\right)}\right] \ldots\right]\right.\right.\right.\right.\right. \\
+\left[a_{1}^{\left(k_{1}\right)},\left[a_{2}^{\left(k_{2}\right)}, \ldots,\left[\widehat{a_{i}^{\left(k_{i}\right)}}, \ldots,\left[a_{s-1}^{\left(k_{s-1}\right)},\left[a_{i}^{\left(k_{i}\right)}, v a_{s}^{\left(k_{s}\right)}\right]\right] \ldots\right]\right)\right. \\
=\frac{\zeta^{\sum_{i=1}^{s} k_{i}}-1}{\zeta^{k_{s}}-1}\left[a_{1}^{\left(k_{1}\right)},\left[a_{2}^{\left(k_{2}\right)}, \ldots,\left[a_{s-1}^{\left(k_{s-1}\right)}, v a_{s}^{\left(k_{s}\right)}\right] \ldots\right]\right.
\end{array}
$$

and the lemma again follows.

The only case we have not considered yet is when all $1 \leqslant k_{i} \leqslant m-1$ and $m \mid \sum_{i=2}^{s} k_{i}$. But then $m \mid\left(k_{2}+\sum_{i=3}^{s} k_{i}\right)$ and we can apply Lemma 4.5 .

$$
\begin{array}{r}
v\left[a_{1}^{\left(k_{1}\right)},\left[a_{2}^{\left(k_{2}\right)},\left[a_{3}^{\left(k_{3}\right)} \ldots,\left[a_{s-1}^{\left(k_{s-1}\right)}, a_{s}^{\left(k_{s}\right)}\right] \ldots\right]\right.\right. \\
=\frac{\zeta^{\sum_{i=1}^{s} k_{i}}-1}{\zeta^{\sum_{i=3}^{s} k_{i}}-1}\left[a_{1}^{\left(k_{1}\right)},\left[a_{2}^{\left(k_{2}\right)}, v\left[a_{3}^{\left(k_{3}\right)} \ldots,\left[a_{s-1}^{\left(k_{s-1}\right)}, a_{s}^{\left(k_{s}\right)}\right] \ldots\right]\right]\right]
\end{array}
$$

and the lemma follows by the induction assumption for $s-2$.

Lemma 4.7. Let $L$ be an $H_{m^{2}}(\zeta)$-module Lie algebra over a field $F$ of characteristic char $F \nmid$ $m$, char $F \neq 2$. Suppose $L$ is a $\mathbb{Z}_{m}$-graded simple Lie algebra, $v L \neq 0$. Then $\operatorname{ker} v=L^{(0)}$.

Proof. By Lemma 4.4. $v L^{(0)}=0$. Since $v c=\zeta c v$, ker $v$ is a $\mathbb{Z}_{m}$-graded subspace. Suppose ker $v \supsetneqq L^{(0)}$. Then there exists an element $a^{(k)} \subseteq L^{(k)}, 1 \leqslant k \leqslant m-1$, $a^{(k)} \neq 0, v a^{(k)}=0$. Since $L$ is $\mathbb{Z}_{m}$-graded simple and $a^{(k)}$ is homogeneous, we have

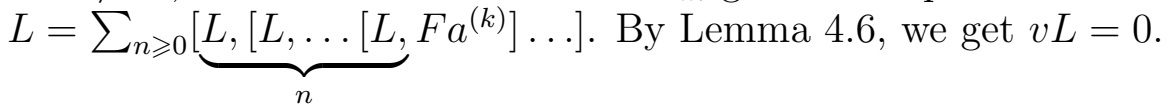

Lemma 4.8. Let $L$ be an $H_{m^{2}}(\zeta)$-module Lie algebra over a field $F$ of characteristic char $F \nmid$ $m$. Suppose $L$ is a $\mathbb{Z}_{m}$-graded simple Lie algebra, $v L \neq 0$. Then $v L^{(k)}=L^{(k-1)}$ for all $1 \leqslant k \leqslant m-1$.

Proof. First, we claim that $L=v L+\sum_{k \neq 1}^{m-1}\left[L^{(k)}, v L^{(m-k)}\right]$. Note that

$$
I=v L+\sum_{k=1}^{m-1}\left[L^{(k)}, v L^{(m-k)}\right]
$$

is a $\mathbb{Z}_{m}$-graded subspace. We claim that $I$ is an ideal too.

By (4.4), $\left[L^{(\ell)}, v L^{(k)}\right] \subseteq v L$ for all $0 \leqslant k, \ell<m$ such that $m \nmid(k+\ell)$. Hence $[L, v L] \subseteq I$.

Now we show that $\left[L, \sum_{k=1}^{m-1}\left[L^{(k)}, v L^{(m-k)}\right]\right] \subseteq I$. 
First, the Jacobi identity implies

$$
\left[a^{(\ell)},\left[b^{(k)}, v u^{(m-k)}\right]\right]=\left[\left[a^{(\ell)}, b^{(k)}\right], v u^{(m-k)}\right]+\left[b^{(k)},\left[a^{(\ell)}, v u^{(m-k)}\right]\right]
$$

for all $0 \leqslant k, \ell<m$ and $a^{(\ell)} \in L^{(\ell)}, b^{(k)} \in L^{(k)}, u^{(m-k)} \in L^{(m-k)}$.

If $\ell=0$, then by (4.4) and (4.6),

$$
\left[a^{(\ell)},\left[b^{(k)}, v u^{(m-k)}\right]\right]=\left[\left[a^{(\ell)}, b^{(k)}\right], v u^{(m-k)}\right]+\left[b^{(k)}, v\left[a^{(\ell)}, u^{(m-k)}\right]\right] \in\left[L^{(k)}, v L^{(m-k)}\right] .
$$

If $\ell \neq 0$ and $k \neq \ell$, then by (4.4) and (4.6),

$$
\left[a^{(\ell)},\left[b^{(k)}, v u^{(m-k)}\right]\right]=\frac{\zeta^{m-k}-1}{\zeta^{\ell}-1} v\left(\left[\left[a^{(\ell)}, b^{(k)}\right], u^{(m-k)}\right]+\left[b^{(k)},\left[a^{(\ell)}, u^{(m-k)}\right]\right]\right) \in v L .
$$

Suppose $\ell \neq 0$ and $k=\ell$. Below we show that $\left[L^{(k)},\left[L^{(k)}, v L^{(m-k)}\right]\right] \subseteq v L$.

If $m \neq 2 k$, then $m-k \neq k$. By 4.3, 4.4 and the Jacobi identity,

$$
\begin{array}{r}
{\left[L^{(k)},\left[L^{(k)}, v L^{(m-k)}\right]\right] \subseteq\left[L^{(k)},\left[L^{(m-k)}, v L^{(k)}\right]\right]} \\
\subseteq\left[\left[L^{(k)}, L^{(m-k)}\right], v L^{(k)}\right]+\left[L^{(m-k)},\left[L^{(k)}, v L^{(k)}\right]\right] \subseteq v L .
\end{array}
$$

If $m=2 k$, then char $F \neq 2$ and the inclusion $\left[L^{(k)},\left[L^{(k)}, v L^{(k)}\right]\right] \subseteq v L$ is a consequence of Lemma 4.5 .

Thus $I$ is indeed a $\mathbb{Z}_{m}$-graded ideal and $L=v L+\sum_{k \neq 0}\left[L^{(k)}, v L^{(m-k)}\right]$ since $L$ is $\mathbb{Z}_{m}$-graded simple.

Since $v c=\zeta c v$, we have $v L^{(k)} \subseteq L^{(k-1)}$, and $\sum_{k \neq 0}\left[L^{(k)}, v L^{(m-k)}\right] \subseteq L^{(m-1)}$. Thus $\bigoplus_{k=0}^{m-2} L^{(k)} \subseteq v L$. Since by Lemma 4.4 we have $v L^{(0)}=0$, this implies $v L \cap L^{(m-1)}=0$ and $v L=\bigoplus_{k=0}^{m-2} L^{(k)}$. In particular, $v L^{(k)}=L^{(k-1)}$ for all $1 \leqslant k \leqslant m-1$.

Lemma 4.9. Let $L$ be an $H_{m^{2}}(\zeta)$-module Lie algebra over a field $F$ of characteristic char $F \nmid$ $m$, char $F \neq 2$. Suppose $L$ is a $\mathbb{Z}_{m}$-graded simple Lie algebra, $v L \neq 0$. Define the maps $\varphi: L^{(k)} \rightarrow L^{(k+1)}$ (we denote them by the same letter) by $\varphi(v a)=a$ for $a \in L^{(k+1)}, 0 \leqslant k \leqslant$ $m-2$. Define $\{a, b\}:=(m-1) !_{\zeta}\left[\varphi(a), \varphi^{m-1}(b)\right]$. Then

$$
\left[\varphi^{k}(a), \varphi^{\ell}(b)\right]:=\left\{\begin{aligned}
&\left(\begin{array}{c}
k+\ell \\
k
\end{array}\right)_{\zeta} \varphi^{k+\ell}[a, b] \text { if } \quad k+\ell<m, \\
& \frac{(k+\ell-m) !_{\zeta}}{k !_{\zeta} !_{\zeta}} \varphi^{k+\ell-m}\{a, b\} \quad \text { if } \quad k+\ell \geqslant m
\end{aligned}\right.
$$

for all $a, b \in L^{(0)}$ and $0 \leqslant k, \ell<m$.

Proof. By Lemmas 4.7 and 4.8, $\varphi$ is well-defined. Moreover, $v \varphi(a)=a$ for all $a \in L^{(i)}$, $0 \leqslant i \leqslant m-2$.

If $k=\ell=0$, then (4.7) is trivial. By Lemma 4.4, $v\left[\varphi^{k}(a), b\right]=\left[\varphi^{k-1}(a), b\right]$ for $1 \leqslant k \leqslant$ $m-1$ and $a, b \in L^{(0)}$. Hence $\left[\varphi^{k}(a), b\right]=\varphi^{k}[a, b]$ and we get 4.7) in the case when at least one of $k, \ell$ is zero.

The case of arbitrary $k, \ell>0, k+\ell<m$, is done by induction using 4.2):

$$
\begin{array}{r}
{\left[\varphi^{k}(a), \varphi^{\ell}(b)\right]=\varphi\left(v\left[\varphi^{k}(a), \varphi^{\ell}(b)\right]\right)=\varphi\left(\left[c \varphi^{k}(a), \varphi^{\ell-1}(b)\right]+\left[\varphi^{k-1}(a), \varphi^{\ell}(b)\right]\right)} \\
=\varphi\left(\left[\zeta^{k} \varphi^{k}(a), \varphi^{\ell-1}(b)\right]+\left[\varphi^{k-1}(a), \varphi^{\ell}(b)\right]\right) \\
=\varphi\left(\zeta^{k}\left(\begin{array}{c}
k+\ell-1 \\
k
\end{array}\right)_{\zeta} \varphi^{k+\ell-1}[a, b]+\left(\begin{array}{c}
k+\ell-1 \\
k-1
\end{array}\right)_{\zeta} \varphi^{k+\ell-1}[a, b]\right) \\
=\left(\zeta^{k}\left(\begin{array}{c}
k+\ell-1 \\
k
\end{array}\right)_{\zeta}+\left(\begin{array}{c}
k+\ell-1 \\
k-1
\end{array}\right)_{\zeta} \varphi^{k+\ell}[a, b]\right. \\
=\left(\begin{array}{c}
k+\ell \\
k
\end{array}\right)_{\zeta} \varphi^{k+\ell}[a, b] .
\end{array}
$$


Suppose $k+\ell=m$. We prove the assertion by induction on $k$. If $k=1$ and $\ell=m-1$, then (4.7) follows from the definition of $\{$, $\}$. If $k>1$, then $\ell<m-1$ and by (4.3) and the induction assumption for $k-1$, we have

$$
\begin{aligned}
{\left[\varphi^{k}(a), \varphi^{\ell}(b)\right] } & =\left[\varphi^{k}(a), v \varphi^{\ell+1}(b)\right]=\frac{\zeta^{\ell+1}-1}{\zeta^{k}-1}\left[v \varphi^{k}(a), \varphi^{\ell+1}(b)\right]=\frac{\zeta^{\ell+1}-1}{\zeta^{k}-1}\left[\varphi^{k-1}(a), \varphi^{\ell+1}(b)\right] \\
= & \frac{\zeta^{\ell+1}-1}{\left(\zeta^{k}-1\right)(k-1) !_{\zeta}(\ell+1) !_{\zeta}}\{a, b\}=\frac{(\ell+1)_{\zeta}}{k_{\zeta}(k-1) !_{\zeta}(\ell+1) !_{\zeta}}\{a, b\}=\frac{1}{k !_{\zeta} \ell !_{\zeta}}\{a, b\} .
\end{aligned}
$$

If $k+\ell>m$, then we again use induction on $(k+\ell)$ :

$$
\begin{array}{r}
{\left[\varphi^{k}(a), \varphi^{\ell}(b)\right]=\varphi\left(v\left[\varphi^{k}(a), \varphi^{\ell}(b)\right]\right)=\varphi\left(\left[c \varphi^{k}(a), \varphi^{\ell-1}(b)\right]+\left[\varphi^{k-1}(a), \varphi^{\ell}(b)\right]\right)} \\
=\varphi\left(\left[\zeta^{k} \varphi^{k}(a), \varphi^{\ell-1}(b)\right]+\left[\varphi^{k-1}(a), \varphi^{\ell}(b)\right]\right) \\
=\varphi\left(\zeta^{k} \frac{(k+\ell-m-1) !_{\zeta}}{k !_{\zeta}(\ell-1) !_{\zeta}} \varphi^{k+\ell-m-1}\{a, b\}+\frac{(k+\ell-m-1) !_{\zeta}}{(k-1) !_{\zeta} \ell !_{\zeta}} \varphi^{k+\ell-m-1}\{a, b\}\right) \\
=\left(\zeta^{k} \frac{(k+\ell-m-1) !_{\zeta}}{k !_{\zeta}(\ell-1) !_{\zeta}}+\frac{(k+\ell-m-1) !_{\zeta}}{(k-1) !_{\zeta} \ell !_{\zeta}}\right) \varphi^{k+\ell-m}\{a, b\} \\
=\frac{(k+\ell)_{\zeta}(k+\ell-m-1) !_{\zeta}}{k !_{\zeta} \ell !_{\zeta}} \varphi^{k+\ell-m}\{a, b\}=\frac{(k+\ell-m) !_{\zeta}}{k !_{\zeta} \ell !_{\zeta}} \varphi^{k+\ell-m}\{a, b\}
\end{array}
$$

since $(k+\ell)_{\zeta}=(k+\ell-m)_{\zeta}$ for $m<k+\ell<2 m$.

Lemma 4.10. Let $L$ be a finite dimensional $H_{m^{2}}(\zeta)$-module Lie algebra over an algebraically closed field $F$ of characteristic 0 . Suppose $L$ is a $\mathbb{Z}_{m}$-graded simple Lie algebra, $v L \neq 0$. Then $L^{(0)}$ is a simple Lie algebra and there exist $\gamma \in F, \gamma \neq 0$, such that $\{a, b\}=\gamma[a, b]$ for all $a, b \in L^{(0)}$. In other words, $L \cong L\left(L^{(0)}, \gamma\right)$ as an $H_{m^{2}}(\zeta)$-module Lie algebra.

Proof. Note that by the Jacobi identity we have

$$
\left[\varphi^{m-1}(a), \varphi(b), u\right]+\left[\varphi(b), u, \varphi^{m-1}(a)\right]+\left[u, \varphi^{m-1}(a), \varphi(b)\right]=0
$$

for all $a, b, u \in L^{(0)}$. Together with Lemma 4.9 this implies

$$
[\{a, b\}, u]+\{[b, u], a\}+\{[u, a], b\}=0 .
$$

Again, by the Jacobi identity we have

$$
\left[\varphi^{m-1}(a), \varphi^{m-1}(b), \varphi(u)\right]+\left[\varphi^{m-1}(b), \varphi(u), \varphi^{m-1}(a)\right]+\left[\varphi(u), \varphi^{m-1}(a), \varphi^{m-1}(b)\right]=0
$$

for all $a, b, u \in L^{(0)}$. Together with Lemma 4.9 this implies

$$
[\{a, b\}, u]+[\{b, u\}, a]+[\{u, a\}, b]=0 .
$$

Now (4.8) implies

$$
\begin{aligned}
& {[\{b, u\}, a]+\{[u, a], b\}+\{[a, b], u\}=0,} \\
& {[\{u, a\}, b]+\{[a, b], u\}+\{[b, u], a\}=0 .}
\end{aligned}
$$

Summing this up with (4.8) and using (4.9) and char $F \neq 2$, we get

$$
\{[a, b], u\}+\{[b, u], a\}+\{[u, a], b\}=0
$$

for all $a, b, u \in L^{(0)}$. Together with 4.8 this implies

$$
\{[a, b], u\}=[\{a, b\}, u] .
$$

By Lemma 4.9, $\{a, b\}=-\{b, a\}$. Together with (4.9) and (4.10) this implies

$$
\{[a, b], u\}=[\{a, u\}, b]+[a,\{b, u\}]
$$

for all $a, b, u \in L^{(0)}$. In other words, $\{\cdot, u\}$ is a derivation for all $u \in L^{(0)}$. 
Now we show that $L^{(0)}$ is a simple Lie algebra. Suppose first $I \neq 0$ is an ideal of $L^{(0)}$ such that $\{I, u\} \subseteq I$ for all $u \in L^{(0)}$. Then by Lemma 4.9. $\bigoplus_{k=0}^{m-1} \varphi^{k}(I)$ is a nonzero $\mathbb{Z}_{m}$-graded ideal of $L$. Hence $L=\bigoplus_{k=0}^{m-1} \varphi^{k}(I)$ and $I=L^{(0)}$. By [16, Chapter III, Section 6, Theorem 7], the solvable radical of $L^{(0)}$ is invariant under all derivations. Hence $L^{(0)}$ is semisimple. If $L^{(0)}$ is non-simple, then $L^{(0)}=I_{1} \oplus I_{2}$ for some nonzero ideals $I_{1}$ and $I_{2}$. Let $\delta$ be a derivation of $L^{(0)}$. Then $\delta\left(I_{i}\right)=\delta\left[I_{i}, I_{i}\right] \subseteq\left[\delta\left(I_{i}\right), I_{i}\right]+\left[I_{i}, \delta\left(I_{i}\right)\right] \subseteq I_{i}$. In other words, $I_{i}, i=1,2$, are invariant under all derivations and $L^{(0)}=I_{1}=I_{2}$, i.e. we get a contradiction. Therefore, $L^{(0)}$ is a simple Lie algebra.

Since $L^{(0)}$ is simple, all derivations of $L^{(0)}$ are inner. Hence there exists an $F$-linear map $\psi: L^{(0)} \rightarrow L^{(0)}$ such that $\{a, b\}=[a, \psi(b)]$ for all $a, b \in L^{(0)}$. Now 4.10 implies

$$
[\psi[a, b], u]=\{[a, b], u\}=[\{a, b\}, u]=[[a, \psi(b)], u]
$$

for all $a, b, u \in L^{(0)}$. Since $L^{(0)}$ has zero center, we have $\psi[a, b]=[a, \psi(b)]$ for all $a, b \in L^{(0)}$. In other words, $\psi: L^{(0)} \rightarrow L^{(0)}$ is a homomorphism of $L^{(0)}$-modules. Since $L^{(0)}$ is an irreducible $L^{(0)}$-module, $\psi$ is a scalar map and $\{a, b\}=\gamma[a, b]$ for some $\gamma \in F$. By Lemma 4.9. $L \cong L\left(L^{(0)}, \gamma\right)$ as an $H_{m^{2}}(\zeta)$-module Lie algebra. Since $L$ is $\mathbb{Z}_{m}$-graded simple and, therefore, semisimple, we have $\gamma \neq 0$.

In Theorem 4.11 below we show that each finite dimensional $H_{m^{2}}(\zeta)$-module Lie algebra simple in the ordinary sense is just a $\mathbb{Z}_{m}$-graded Lie algebra with the trivial $v$-action.

Theorem 4.11. Let $L$ be a finite dimensional $H_{m^{2}}(\zeta)$-module Lie algebra over an algebraically closed field $F$ of characteristic 0 . Suppose $L$ is simple in the ordinary sense. Then $v L=0$.

Proof. Suppose $v L \neq 0$. Then by Lemma 4.10 we have the isomorphism $L \cong L\left(L^{(0)}, \gamma\right)$ of $H_{m^{2}}(\zeta)$-module Lie algebras for some $\gamma \neq 0$. By Theorem 4.3 . $L\left(L^{(0)}, \gamma\right) \cong L_{\alpha}\left(L^{(0)}\right)$ where $\alpha=\frac{(1-\zeta)^{-1}}{\sqrt[m]{\gamma}}$. However $L_{\alpha}\left(L^{(0)}\right)$ is non-simple as an ordinary Lie algebra and we get a contradiction. Thus $v L=0$.

\section{Non-SEMISIMPLE $H_{m^{2}}(\zeta)$-SIMPLE LiE ALGEBRAS}

In this section we show that all non-semisimple $H_{m^{2}}(\zeta)$-simple Lie algebras are isomorphic to Lie algebras from Theorem 4.1 with $\gamma=0$.

Theorem 5.1. Suppose $L$ is a finite dimensional $H_{m^{2}}(\zeta)$-simple Lie algebra over an algebraically closed field $F$ of characteristic 0 and the solvable radical of $L$ is nonzero. Then $L$ is isomorphic as an $H_{m^{2}}(\zeta)$-module Lie algebra to the Lie algebra $L(B, 0)$ for some finite dimensional simple Lie algebra $B$.

In order to prove Theorem 5.1, we need several auxiliary lemmas.

Let $M_{1}, M_{2}$ be two $\mathbb{Z}_{m}$-graded modules over a $\mathbb{Z}_{m}$-graded Lie algebra $L$. We say that a $F$-linear bijection $\varphi: M_{1} \underset{\rightarrow}{\rightarrow} M_{2}$ is a c-isomorphism of $M_{1}$ and $M_{2}$ if there exists $r \in \mathbb{Z}$ such that $c \varphi(b)=\zeta^{-r} \varphi(c b), \varphi(a b)=a \varphi(b)$ for all $b \in M_{1}, a \in L$.

Recall that for any finite-dimensional Lie algebra $L$ over a field of characteristic 0 we have $[L, R] \subseteq N$ (see e.g. [15, Proposition 2.1.7]) where $R, N$ are, respectively, the solvable and the nilpotent radical. Hence if $N=0$, then we have $R \subseteq Z(L) \subseteq N=0$ where $Z(L)$ is the center of $L$. Recall also that if $L$ is an $H_{m^{2}}(\zeta)$-module Lie algebra, then $R$ and $N$ are $\mathbb{Z}_{m^{-}}$ graded ideals since $R$ and $N$ are invariant under all automorphisms of $L$ and, in particular, under the $c$-action.

Lemma 5.2. Suppose $L$ is a finite dimensional $H_{m^{2}}(\zeta)$-simple Lie algebra over a field $F$ and its solvable radical $R \neq 0$. Let $N$ be the nilpotent radical of $L, N^{\ell}=0, N^{\ell-1} \neq 0$. Choose a minimal $\mathbb{Z}_{m}$-graded L-ideal $\tilde{N} \subseteq N^{\ell-1}$. Then for any $k$ the subspace $N_{k}:=\sum_{i=0}^{i=k} v^{i} \tilde{N}$ is 
a $\mathbb{Z}_{m}$-graded ideal of $L$ and $L=\bigoplus_{i=0}^{t} v^{i} \tilde{N}$ (direct sum of $\mathbb{Z}_{m}$-graded subspaces) for some $1 \leqslant t \leqslant m-1$. Moreover, $N_{k} / N_{k-1}, 0 \leqslant k \leqslant t$, are irreducible $\mathbb{Z}_{m}$-graded L-modules c-isomorphic to each other. (Here $N_{-1}:=0$.)

Proof. Since for any $a \in \tilde{N}$ and $b \in L$ the element $\left[v^{k} a, b\right]=v\left[v^{k-1} a, b\right]-\left[c v^{k-1} a, v b\right]$ can be presented as an $F$-linear combination of elements $v^{i}\left[c^{k-i} a, v^{k-i} b\right]$, each $N_{k}:=\sum_{i=0}^{i=k} v^{i} \tilde{N}$ is a $\mathbb{Z}_{m}$-graded ideal of $L$.

Recall that $v^{m}=0$. Thus $N_{m-1}$ is an $H_{m^{2}}(\zeta)$-invariant ideal of $L$. Hence $L=N_{m-1}$.

Let $\varphi_{k}: N_{k} / N_{k-1} \rightarrow N_{k+1} / N_{k}$, where $0 \leqslant k \leqslant m-2$, be the map defined by

$$
\varphi_{k}\left(b+N_{k-1}\right)=v b+N_{k} .
$$

Denote $\bar{b}:=b+N_{k-1}$. Then $c \varphi_{k}(\bar{b})=\zeta^{-1} \varphi_{k}(c \bar{b})$,

$$
\begin{gathered}
\varphi_{k}(a \bar{b})=v[a, b]+N_{k}=-v[b, a]+N_{k}=-[c b, v a]-[v b, a]+N_{k} \\
=-[v b, a]+N_{k}=[a, v b]+N_{k}=a \varphi_{k}(\bar{b}) \text { for all } a \in L, b \in N_{k} .
\end{gathered}
$$

Note that $\tilde{N}=N_{0} / N_{-1}$ is an irreducible $\mathbb{Z}_{m}$-graded $L$-module. Therefore, $N_{k+1} / N_{k}=$ $\varphi_{k}\left(N_{k} / N_{k-1}\right)$ is an irreducible $\mathbb{Z}_{m}$-graded $L$-module or zero for any $0 \leqslant k<m-1$. Thus if $L=N_{t}, L \neq N_{t-1}$, then $\operatorname{dim} N_{t}=(t+1) \operatorname{dim} \tilde{N}$ and $L=\bigoplus_{i=0}^{t} v^{i} \tilde{N}$ (direct sum of $\mathbb{Z}_{m}$-graded subspaces).

Lemma 5.3. Assume that we are under the conditions of Lemma 5.2. In addition, suppose that the field $F$ is algebraically closed of characteristic 0 . Then $R=N=N_{t-1}, L^{(i)}=v^{t-i} \tilde{N}$ for $0 \leqslant i \leqslant t$, and $L^{(0)} \cong L / R$ is a simple Lie algebra. Moreover $\operatorname{dim}\left(N_{k} / N_{k-1}\right)=\operatorname{dim}(L / R)$ for all $0 \leqslant k \leqslant t$. In addition, $\operatorname{ker} v=L^{(0)}$.

Proof. First we notice that $[L, L]$ is an $H_{m^{2}}(\zeta)$-invariant ideal. Hence $L=[L, L]$ and $L \neq R$.

By [26], there exists a maximal $\mathbb{Z}_{m}$-graded semisimple Lie subalgebra $B \subseteq L$ such that $L=B \oplus R$ (direct sum of $\mathbb{Z}_{m}$-graded subspaces), $B \cong L / R$. Note that $N$ annihilates all irreducible $\mathbb{Z}_{m}$-graded $L$-modules that are factors of the adjoint representation of $L$. In addition, $[L, R] \subseteq N$ (see e.g. [15, Proposition 2.1.7]). Hence $L / N$ is a reductive $\mathbb{Z}_{m}$-graded Lie algebra and $\tilde{N}$ is an irreducible $\mathbb{Z}_{m}$-graded $L / N$-module. By [9, Lemma 6], we have $\tilde{N}=\bigoplus_{i=0}^{s} c^{i} M$ where $M \subseteq \tilde{N}$ is an $L$-submodule such that $R$ is acting on $M$ by scalar operators, $s \in \mathbb{N}$. Since $\tilde{N}$ is an irreducible $\mathbb{Z}_{m}$-graded $L$-module, we may assume that $M$ is an irreducible $L$-module. All $B$-submodules in $M$ are $L$-submodules since $R$ is acting on $M$ by scalar operators. Hence $M$ is an irreducible $B$-module.

Since $\tilde{N}=N_{0} / N_{-1}$, all $N_{k} / N_{k-1}$ are $c$-isomorphic to each other, and $B$ is semisimple, the Lie algebra $L$ is a direct sum of irreducible $B$-submodules isomorphic to $c^{j} M$ where $j \in \mathbb{Z}$. Note that the $B$-action on each $M$ and therefore on each $c^{j} M$ must be nonzero, since $B$ itself is a $B$-submodule of $L$ with a nonzero $B$-action. On the other hand, there exists a $B$ submodule $Q \subseteq R$ such that $R=N \oplus Q$. Since $[L, R] \subseteq N$, we have $[B, Q] \subseteq N \cap Q=0$, i.e. $Q \subseteq L$ is a submodule with the zero $B$-action. Hence $Q=0$ and $R=N$. In particular, all $N_{k} / N_{k-1}$ are irreducible $\mathbb{Z}_{m}$-graded $B$-modules $c$-isomorphic to each other. However, $B \subseteq L$ is a $\mathbb{Z}_{m}$-graded $B$-submodule. If $B$ is not a $\mathbb{Z}_{m}$-graded simple Lie algebra, then $B$ is a direct sum of $\mathbb{Z}_{m}$-graded simple Lie subalgebras (this follows e.g. from [10, Theorem 9]), which are non- $c$-isomorphic as $B$-modules. Hence $B$ must be a $\mathbb{Z}_{m}$-graded simple Lie algebra and all $N_{k} / N_{k-1}$ are $c$-isomorphic to $B$ as $\mathbb{Z}_{m}$-graded modules. Let $B \subseteq N_{q}, B \varsubsetneqq N_{q-1}$ for some $q \in \mathbb{Z}_{+}$. If $q<t$, then $\left[B, N_{t}\right] \subseteq N_{t-1}$ and $N_{t} / N_{t-1}$ has the zero $B$-action. Since all $N_{k} / N_{k-1}$ are $c$-isomorphic, we get a contradiction. Therefore, $B \cap N_{t-1}=0, L=B \oplus N_{t-1}$ (direct sum of subspaces), and $B \cong L / N_{t-1}$.

We claim that $c a=a$ for all $a \in B$ and therefore $B$ is simple as an ordinary Lie algebra. In Lemma 5.2 we proved that $\varphi_{k}(a \bar{b})=a \varphi_{k}(\bar{b})$ for all $0 \leqslant k \leqslant m-1$ and $a \in L, b \in N_{k}$. 
Analogously, one shows that

$$
\varphi_{k}(a \bar{b})=v[a, b]+N_{k}=[c a, v b]+[v a, b]+N_{k}=[c a, v b]+N_{k}=(c a) \varphi_{k}(\bar{b}) .
$$

In other words, $((c a)-a)$ is acting as 0 on all $N_{k} / N_{k-1}$ for every $a \in B$. In particular, $((c a)-a)$ belongs to the center of $B$. Since $B$ is semisimple, we get $c a=a$ for all $a \in B$ and $B \subseteq L^{(0)}$ has a trivial grading. Hence $B$ is simple as an ordinary Lie algebra.

Note that $B \cong L / N_{t-1} \cong v^{t} \tilde{N}$ as $\mathbb{Z}_{m}$-graded spaces. Hence $v^{t} \tilde{N} \subseteq L^{(0)}$. Using $v c=\zeta c v$, we get $v^{i} \tilde{N} \subseteq L^{(t-i)}$. Since $L=\bigoplus_{i=0}^{m-1} L^{(i)}=\bigoplus_{i=0}^{t} v^{i} \tilde{N}$, we obtain $L^{(i)}=v^{t-i} \tilde{N}$ for $0 \leqslant i \leqslant t$, $L^{(i)}=0$ for $t+1 \leqslant i \leqslant m-1$. In particular, $B=L^{(0)}=v^{t} \tilde{N}$.

Recall that each $N_{j}=\bigoplus_{i=0}^{j} v^{i} \tilde{N}=\bigoplus_{i=t-j}^{t} L^{(i)}$ is an ideal. Hence for $0 \leqslant j \leqslant t$ and $0 \leqslant i \leqslant m-1$ we always have $0 \leqslant t-j+i<t-j+m$,

$$
\left[L^{(i)}, L^{(t-j)}\right] \subseteq N_{j} \cap L^{(t-j+i)} \subseteq N_{j-i},
$$

and $\left[L^{(i)}, N_{k}\right] \subseteq N_{k-i}$. (We assume that $N_{k}:=0$ for $k<0$.) In particular, the ideal $N_{t-1}$ is nilpotent and $R=N=N_{t-1}$.

If $t=m-1$, then $v L^{(0)}=v^{m} \tilde{N}=0$. Since $N_{t-1} \cap(\operatorname{ker} v)=0$, we get $\operatorname{ker} v=L^{(0)}$. If $t<m-1$, then $v L^{(0)} \subseteq L^{(m-1)}=0$. Again, $\operatorname{ker} v=L^{(0)}$.

Lemma 5.4. Suppose we are under the assumptions of Lemma 5.3. Define the F-linear $\operatorname{map} \varphi: L \rightarrow L$ by $\varphi\left(v^{k} a\right)=v^{k-1}$ a for all $a \in \tilde{N}, 1 \leqslant k \leqslant t, \varphi(\tilde{N})=0$. Then

$$
\left[\varphi^{k}(a), \varphi^{\ell}(b)\right]=\left(\begin{array}{c}
k+\ell \\
k
\end{array}\right)_{\zeta} \varphi^{k+\ell}[a, b] \text { for all } a, b \in L^{(0)} \text { and } 0 \leqslant k, \ell \leqslant t .
$$

Proof. Note that $\varphi(v a)=a$ for all $a \in N_{t-1}$. Thus for every $b \in L^{(0)}, a=v u, u \in N_{t-1}$ we have

$$
\begin{gathered}
c \varphi(a)=c \varphi(v u)=c u=\varphi(v c u)=\zeta \varphi(c v u)=\zeta \varphi(c a), \\
\varphi[a, b]=\varphi[v u, b]=\varphi(v[u, b]-[c u, v b])=[u, b]=[\varphi(a), b] .
\end{gathered}
$$

Since $\varphi(\tilde{N})=0, L=v N_{t-1} \oplus \tilde{N}$ (direct sum of $\mathbb{Z}_{m}$-graded subspaces), and $\tilde{N}$ is an ideal, we have $c \varphi(a)=\zeta \varphi(c a), \varphi[a, b]=[\varphi(a), b]$ for all $a \in L, b \in L^{(0)}$. This proves (5.1) for $k=0$ or $\ell=0$.

The case of arbitrary $k, \ell \geqslant 1$ is done by induction using

$$
\left[\varphi^{k}(a), \varphi^{\ell}(b)\right]=\varphi\left(v\left[\varphi^{k}(a), \varphi^{\ell}(b)\right]\right)=\varphi\left(\left[c \varphi^{k}(a), \varphi^{\ell-1}(b)\right]+\left[\varphi^{k-1}(a), \varphi^{\ell}(b)\right]\right)
$$

analogously to Lemma 4.9 .

Proof of Theorem 5.1. By Lemma 5.3, $L^{(0)}$ is a simple Lie algebra. Let $a, b \in L^{(0)}$ such that $[a, b] \neq 0$. Then $\varphi^{t}[a, b] \neq 0$.

Note that $\left[\varphi^{t}(a), \varphi(b)\right]=\left(\begin{array}{c}t+1 \\ t\end{array}\right)_{\zeta} \varphi^{t+1}[a, b]=0$. However

$$
\begin{aligned}
& 0=v\left[\varphi^{t}(a), \varphi(b)\right]=\left[v \varphi^{t}(a), \varphi(b)\right]+\left[c \varphi^{t}(a), v \varphi(b)\right] \\
& =\left(\left(\begin{array}{c}
t \\
t-1
\end{array}\right)_{\zeta}+\zeta^{t}\right) \varphi^{t}[a, b]=(t+1)_{\zeta} \varphi^{t}[a, b] .
\end{aligned}
$$

Hence $(t+1)_{\zeta}=0$ and $m=t+1$. Now (5.1) and Lemma 5.3 imply the theorem.

Remark 5.5. Since the maximal semisimple Lie subalgebra ker $v$ is uniquely determined, any two such $H_{m^{2}}(\zeta)$-simple Lie algebras $L$ are isomorphic as $H_{m^{2}}(\zeta)$-module Lie algebras if and only if their Lie subalgebras ker $v$ are isomorphic as ordinary algebras. Moreover, all automorphisms of $L$ as an $H_{m^{2}}(\zeta)$-module Lie algebra are induced by the automorphisms of ker $v$ as a Lie algebra. Indeed, let $\psi: L \widetilde{\Im} L$ be an automorphism of $L$ as an $H_{m^{2}}(\zeta)$-module Lie algebra. Since $\tilde{N}=N^{m-1}$, we have $\psi(\tilde{N})=\tilde{N}$ and $\psi\left(v^{k} \tilde{N}\right)=v^{k} \tilde{N}$ for all $0 \leqslant k<m$. 
Now $v^{k} \psi\left(\varphi^{k}(a)\right)=\psi(a)$ for all $a \in \operatorname{ker} v$ implies $\psi\left(\varphi^{k}(a)\right)=\varphi^{k}(\psi(a))$ and $\psi$ is uniquely determined by its restriction on ker $v$.

\section{Polynomial $H$-identities}

In Section 7 we prove that if $L$ is a finite dimensional $H_{m^{2}}(\zeta)$-simple $H_{m^{2}}(\zeta)$-module Lie algebra over an algebraically closed field of characteristic 0 , then $\operatorname{PIexp}^{H_{m^{2}}(\zeta)}(L)=\operatorname{dim} L$. In particular, the $H_{m^{2}}(\zeta)$-PI-exponent of $L$ is integer and the analog of Amitsur's conjecture holds for polynomial $H_{m^{2}}(\zeta)$-identities of $L$.

Let $F$ be a field and let $F\{X\}$ be the absolutely free nonassociative algebra on the set $X:=\left\{x_{1}, x_{2}, x_{3}, \ldots\right\}$. Then $F\{X\}=\bigoplus_{n=1}^{\infty} F\{X\}^{(n)}$ where $F\{X\}^{(n)}$ is the $F$-linear span of all monomials of total degree $n$. Let $H$ be a Hopf algebra over a field $F$. Consider the algebra

$$
F\{X \mid H\}:=\bigoplus_{n=1}^{\infty} H^{\otimes n} \otimes F\{X\}^{(n)}
$$

with the multiplication $\left(u_{1} \otimes w_{1}\right)\left(u_{2} \otimes w_{2}\right):=\left(u_{1} \otimes u_{2}\right) \otimes w_{1} w_{2}$ for all $u_{1} \in H^{\otimes j}, u_{2} \in H^{\otimes k}$, $w_{1} \in F\{X\}^{(j)}, w_{2} \in F\{X\}^{(k)}$. We use the notation

$$
x_{i_{1}}^{h_{1}} x_{i_{2}}^{h_{2}} \ldots x_{i_{n}}^{h_{n}}:=\left(h_{1} \otimes h_{2} \otimes \ldots \otimes h_{n}\right) \otimes x_{i_{1}} x_{i_{2}} \ldots x_{i_{n}}
$$

(the arrangements of brackets on $x_{i_{j}}$ and on $x_{i_{j}}^{h_{j}}$ are the same). Here $h_{1} \otimes h_{2} \otimes \ldots \otimes h_{n} \in H^{\otimes n}$, $x_{i_{1}} x_{i_{2}} \ldots x_{i_{n}} \in F\{X\}^{(n)}$.

Note that if $\left(\gamma_{\beta}\right)_{\beta \in \Lambda}$ is a basis in $H$, then $F\{X \mid H\}$ is isomorphic to the absolutely free nonassociative algebra over $F$ with free formal generators $x_{i}^{\gamma_{\beta}}, \beta \in \Lambda, i \in \mathbb{N}$.

Define on $F\{X \mid H\}$ the structure of a left $H$-module by

$$
h\left(x_{i_{1}}^{h_{1}} x_{i_{2}}^{h_{2}} \ldots x_{i_{n}}^{h_{n}}\right)=x_{i_{1}}^{h_{(1)} h_{1}} x_{i_{2}}^{h_{(2)} h_{2}} \ldots x_{i_{n}}^{h_{(n)} h_{n}},
$$

where $h_{(1)} \otimes h_{(2)} \otimes \ldots \otimes h_{(n)}$ is the image of $h$ under the comultiplication $\Delta$ applied $(n-1)$ times, $h \in H$. Then $F\{X \mid H\}$ is the absolutely free $H$-module nonassociative algebra on $X$, i.e. for each map $\psi: X \rightarrow A$ where $A$ is an $H$-module algebra, there exists the unique homomorphism $\bar{\psi}: F\{X \mid H\} \rightarrow A$ of algebras and $H$-modules, such that $\left.\bar{\psi}\right|_{X}=\psi$. Here we identify $X$ with the set $\left\{x_{j}^{1_{H}} \mid j \in \mathbb{N}\right\} \subset F\{X \mid H\}$.

Consider the $H$-invariant ideal $I$ in $F\{X \mid H\}$ generated by the set

$$
\{u(v w)+v(w u)+w(u v) \mid u, v, w \in F\{X \mid H\}\} \cup\left\{u^{2} \mid u \in F\{X \mid H\}\right\} .
$$

Then $L(X \mid H):=F\{X \mid H\} / I$ is the free $H$-module Lie algebra on $X$, i.e. for any $H$-module Lie algebra $L$ and a map $\psi: X \rightarrow L$, there exists the unique homomorphism $\bar{\psi}: L(X \mid H) \rightarrow L$ of Lie algebras and $H$-modules such that $\left.\bar{\psi}\right|_{X}=\psi$. We refer to the elements of $L(X \mid H)$ as Lie H-polynomials.

Remark 6.1. If $H$ is cocommutative and char $F \neq 2$, then $L(X \mid H)$ is the ordinary free Lie algebra with free generators $x_{i}^{\gamma_{\beta}}, \beta \in \Lambda, i \in \mathbb{N}$ where $\left(\gamma_{\beta}\right)_{\beta \in \Lambda}$ is a basis in $H$, since the ordinary ideal of $F\{X \mid H\}$ generated by (6.1) is already $H$-invariant. However, if $h_{(1)} \otimes h_{(2)} \neq$ $h_{(2)} \otimes h_{(1)}$ for some $h \in H$, we still have

$$
\left[x_{i}^{h_{(1)}}, x_{j}^{h_{(2)}}\right]=h\left[x_{i}, x_{j}\right]=-h\left[x_{j}, x_{i}\right]=-\left[x_{j}^{h_{(1)}}, x_{i}^{h_{(2)}}\right]=\left[x_{i}^{h_{(2)}}, x_{j}^{h_{(1)}}\right]
$$

in $L(X \mid H)$ for all $i, j \in \mathbb{N}$, i.e. in the case $h_{(1)} \otimes h_{(2)} \neq h_{(2)} \otimes h_{(1)}$ the Lie algebra $L(X \mid H)$ is not free as an ordinary Lie algebra. 
Let $L$ be an $H$-module Lie algebra for some Hopf algebra $H$ over a field $F$. An $H$-polynomial $f \in L(X \mid H)$ is a $H$-identity of $L$ if $\psi(f)=0$ for all homomorphisms $\psi: L(X \mid H) \rightarrow L$ of Lie algebras and $H$-modules. In other words, $f\left(x_{1}, x_{2}, \ldots, x_{n}\right)$ is a polynomial $H$-identity of $L$ if and only if $f\left(a_{1}, a_{2}, \ldots, a_{n}\right)=0$ for any $a_{i} \in L$. In this case we write $f \equiv 0$. The set $\operatorname{Id}^{H}(L)$ of all polynomial $H$-identities of $L$ is an $H$-invariant ideal of $L(X \mid H)$.

Example 6.2. Note that if $m=2$ and $\zeta=-1$, then $(1-c) L\left(\mathfrak{s l}_{2}(F), 0\right)=L\left(\mathfrak{s l}_{2}(F), 0\right)^{(1)}$ and the commutator of any two elements of $L\left(\mathfrak{s l}_{2}(F), 0\right)^{(1)}$ is zero by (4.1). Hence

$$
\left[x^{1-c}, y^{1-c}\right] \in \operatorname{Id}^{H_{4}(-1)}\left(L\left(\mathfrak{s l}_{2}(F), 0\right)\right) .
$$

Denote by $V_{n}^{H}$ the space of all multilinear Lie $H$-polynomials in $x_{1}, \ldots, x_{n}, n \in \mathbb{N}$, i.e.

$$
V_{n}^{H}=\left\langle\left[x_{\sigma(1)}^{h_{1}}, x_{\sigma(2)}^{h_{2}}, \ldots, x_{\sigma(n)}^{h_{n}}\right] \mid h_{i} \in H, \sigma \in S_{n}\right\rangle_{F} \subset L(X \mid H) .
$$

Then the number $c_{n}^{H}(L):=\operatorname{dim}\left(\frac{V_{n}^{H}}{V_{n}^{H} \cap \mathrm{d}^{H}(L)}\right)$ is called the $n$th codimension of polynomial $H$-identities or the $n$th $H$-codimension of $L$.

Remark 6.3. One can treat polynomial $H$-identities of $L$ as $H$-identities of a nonassociative $H$-module algebra (i.e. use $F\{X \mid H\}$ instead of $L(X \mid H)$ ) and define their codimensions. However those codimensions will coincide with $c_{n}^{H}(L)$ since the $n$th $H$-codimension equals the dimension of the subspace in $\operatorname{Hom}_{F}\left(L^{\otimes n} ; L\right)$ that consists of those $n$-linear functions that can be represented by $H$-polynomials. of $L$.

Recall that the limit PIexp ${ }^{H}(L):=\lim _{n \rightarrow \infty} \sqrt[n]{c_{n}^{H}(L)}$ (if it exists) is called the $H$-PI-exponent

One of the main tools in the investigation of polynomial identities is provided by the representation theory of symmetric groups. The symmetric group $S_{n}$ acts on the space $\frac{V_{n}^{H}}{V_{n}^{H} \cap \mathrm{Id}^{H}(L)}$ by permuting the variables. If the base field $F$ is of characteristic 0 , then irreducible $F S_{n}$-modules are described by partitions $\lambda=\left(\lambda_{1}, \ldots, \lambda_{s}\right) \vdash n$ and their Young diagrams $D_{\lambda}$. The character $\chi_{n}^{H}(L)$ of the $F S_{n}$-module $\frac{V_{n}^{H}}{V_{n}^{H} \cap \mathrm{Id}^{H}(L)}$ is called the $n$th cocharacter of polynomial $H$-identities of $L$. We can rewrite it as a sum

$$
\chi_{n}^{H}(L)=\sum_{\lambda \vdash n} m(L, H, \lambda) \chi(\lambda)
$$

of irreducible characters $\chi(\lambda)$. Let $e_{T_{\lambda}}=a_{T_{\lambda}} b_{T_{\lambda}}$ and $e_{T_{\lambda}}^{*}=b_{T_{\lambda}} a_{T_{\lambda}}$ where $a_{T_{\lambda}}=\sum_{\pi \in R_{T_{\lambda}}} \pi$ and $b_{T_{\lambda}}=\sum_{\sigma \in C_{T_{\lambda}}}(\operatorname{sign} \sigma) \sigma$, be the Young symmetrizers corresponding to a Young tableau $T_{\lambda}$. Then $M(\lambda) \stackrel{F}{=} F S_{n} e_{T_{\lambda}} \cong F S_{n} e_{T_{\lambda}}^{*}$ is an irreducible $F S_{n}$-module corresponding to a partition $\lambda \vdash n$. We refer the reader to [1, 4, 7] for an account of $S_{n}$-representations and their applications to polynomial identities.

\section{Exponent of $H_{m^{2}}(\zeta)$-IDENTITIES OF $H_{m^{2}}(\zeta)$-SIMPle LiE AlGEBRAS}

In this section we prove the existence of the $H_{m^{2}}(\zeta)$-PI-exponent for $H_{m^{2}}(\zeta)$-simple Lie algebras:

Theorem 7.1. Let $L$ be a finite dimensional $H_{m^{2}}(\zeta)$-simple Lie algebra over an algebraically closed field $F$ of characteristic 0 . Then there exist $C>0$ and $r \in \mathbb{R}$ such that

$$
C n^{r}(\operatorname{dim} L)^{n} \leqslant c_{n}^{H_{m^{2}}(\zeta)}(L) \leqslant(\operatorname{dim} L)^{n+1} \text { for all } n \in \mathbb{N} .
$$

In particular, PIexp ${ }^{H^{2}(\zeta)}(L)=\operatorname{dim} L$ and the analog of Amitsur's conjecture holds for $L$.

First we need the following standard observation: 
Lemma 7.2. Let $L$ be a finite dimensional $H$-module Lie algebra over a field of characteristic 0 . Let $\lambda \vdash n, n \in \mathbb{N}$. Suppose $\lambda_{(\operatorname{dim} L)+1}>0$. Then $m(L, H, \lambda)=0$.

Proof. It is sufficient to prove that $e_{T_{\lambda}}^{*} f \in \operatorname{Id}^{H}(L)$ for all $f \in V_{n}^{H}$. Fix some basis of $L$. Since polynomials are multilinear, it is sufficient to substitute only basis elements. Note that $e_{T_{\lambda}}^{*}=b_{T_{\lambda}} a_{T_{\lambda}}$ where $b_{T_{\lambda}}$ alternates the variables of each column of $T_{\lambda}$. Hence if we make a substitution and $e_{T_{\lambda}}^{*} f$ does not vanish, this implies that different basis elements are substituted for the variables of each column. But if $\lambda_{(\operatorname{dim} L)+1}>0$, then the length of the first column is greater than $\operatorname{dim} L$. Therefore, $e_{T_{\lambda}}^{*} f \in \operatorname{Id}^{H}(L)$.

Now we prove the existence of a polynomial $H$-non-identity with many alternations:

Lemma 7.3. Let $L$ be a finite dimensional non-semisimple $H_{m^{2}}(\zeta)$-simple Lie algebra over an algebraically closed field $F$ of characteristic 0. Let $\ell:=\operatorname{dim} L^{(0)}$. Then exists a number $r \in \mathbb{N}$ such that for every $n \geqslant \ell m r+1$ there exist disjoint subsets $X_{1}, \ldots, X_{k r} \subseteq\left\{x_{1}, \ldots, x_{n}\right\}$, $k:=\left[\frac{n-1}{\ell m r}\right],\left|X_{1}\right|=\ldots=\left|X_{k r}\right|=\ell m$ and a polynomial $f \in V_{n}^{H_{m^{2}}(\zeta)} \backslash \operatorname{Id}^{H_{m^{2}}(\zeta)}(L)$ alternating in the variables of each set $X_{j}$.

Proof. Since $L$ is not semisimple, Theorem 5.1 implies $L \cong L(B, 0)$ for some simple Lie algebra $B, \operatorname{dim} B=\ell$. We have the $\mathbb{Z}_{m}$-grading $L=\bigoplus_{k=0}^{m-1} L^{(k)}$ (see Remark 2.3) where $L^{(0)}$ can be identified with $B$. By Yu. P. Razmyslov's theorem [24, Theorem 12.1], there exists $r \in \mathbb{N}$ such that for every $k \in \mathbb{N}$ there exists a multilinear associative polynomial

$$
f_{0}=f_{0}\left(x_{11}, \ldots, x_{1 \ell} ; \ldots ; x_{k r, 1}, \ldots, x_{k r, \ell}\right)
$$

alternating in the variables of each set $\left\{x_{i 1}, \ldots, x_{i \ell}\right\}, 1 \leqslant i \leqslant k r$, such that $f_{0}\left(\operatorname{ad} a_{1}, \ldots, \operatorname{ad} a_{\ell} ; \ldots ;\right.$ ad $a_{1}, \ldots$, ad $\left.a_{\ell}\right)$ is a nonzero scalar operator on $L$ for any basis $a_{1}, \ldots, a_{\ell}$ of $B$. Here $(\operatorname{ad} x) y:=[x, y]$.

Let $n \in \mathbb{N}$. Define $k:=\left[\frac{n-1}{\ell m r}\right]$. Choose a polynomial $f_{0}$ as above alternating in $k r$ sets of $\ell$ variables and a polynomial $\tilde{f}_{0}$ alternating in $m r$ sets of $\ell$ variables. Consider the Lie $H_{m^{2}}(\zeta)$-polynomial

$$
f_{1}:=\tilde{f}_{0}\left(\operatorname{ad} y_{11}, \ldots, \operatorname{ad} y_{1 \ell} ; \ldots ; \operatorname{ad} y_{m r, 1}, \ldots, \operatorname{ad} y_{m r, \ell}\right) f_{2}
$$

where

$$
f_{2}=\left(\prod_{i=1}^{m} f_{0}\left(\operatorname{ad}\left(x_{11 i}^{v^{i-1}}\right), \ldots, \operatorname{ad}\left(x_{1 \ell i}^{v^{i-1}}\right) ; \ldots ; \operatorname{ad}\left(x_{k r, 1, i}^{v^{i-1}}\right), \ldots, \operatorname{ad}\left(x_{k r, \ell, i}^{v^{i-1}}\right)\right)\right) z .
$$

Let $b_{1}, \ldots, b_{\ell}$ be a basis of $L^{(m-1)}$. Then $v^{m-1} b_{1}, \ldots, v^{m-1} b_{\ell}$ is a basis of $L^{(0)}=B$. Hence $f_{1}$ does not vanish under the substitution $x_{j t i}=v^{m-i} b_{t}, y_{j t}=v^{m-1} b_{t}$ and $z=\bar{z}$ for any nonzero $\bar{z} \in B$. Fix some $\bar{z}$ and denote this substitution by $\Xi$. Let $b$ be the value of $f_{1}$ under $\Xi$. Consider $f_{3}:=\operatorname{Alt}_{1} \ldots$ Alt $_{k r} f_{1}$ where Alt $_{j}$ is the operator of alternation in the variables of the set $X_{j}=\left\{x_{j t i} \mid 1 \leqslant t \leqslant \ell, 1 \leqslant i \leqslant m\right\}$. Note that $v^{m}=0$ implies that all the items where $x_{j t i}$ is replaced with some $x_{j t^{\prime} i^{\prime}}$ for $i^{\prime}<i$, vanish. Hence all permutations in the alternations mix variables just in every set $\left\{x_{j t i} \mid 1 \leqslant t \leqslant \ell\right\}$ for fixed $j$ and $i$. Since $f_{1}$ is alternating in the variables of these sets, the value of $f_{3}$ under $\Xi$ equals $(\ell !)^{k m r} b \neq 0$.

Note that $k \ell m r+1 \leqslant n<\operatorname{deg} f_{3}=(k+1) \ell m r+1$. We can expand $\tilde{f}_{0}$ and rewrite $f_{3}$ as a linear combination of

$$
\begin{array}{r}
f_{4}:=\left[w_{1},\left[w_{2}, \ldots,\left[w_{\ell m r}, \operatorname{Alt}_{1} \ldots \operatorname{Alt}_{k r}\left(\prod _ { i = 1 } ^ { m } f _ { 0 } \left(\operatorname{ad}\left(x_{11 i}^{v^{i-1}}\right), \ldots, \operatorname{ad}\left(x_{1 \ell i}^{v^{i-1}}\right)\right.\right.\right.\right.\right. \\
\left.\left.\left.\left.\ldots ; \operatorname{ad}\left(x_{k r, 1, i}^{v^{i-1}}\right), \ldots, \operatorname{ad}\left(x_{k r, \ell, i}^{v^{i-1}}\right)\right)\right) z\right] \ldots\right]
\end{array}
$$


where the variables $w_{i}$ are the variables $y_{j t}$ taken in some order depending on the item. Since $f_{3} \notin \operatorname{Id}^{H_{m^{2}}(\zeta)}(L)$, one of the items $f_{4}$ does not vanish under $\Xi$. Then

$$
\begin{array}{r}
f=\left[w_{(k+1) \ell m r-n+2},\left[w_{(k+1) \ell m r-n+3}, \ldots,\left[w_{\ell m r}, \operatorname{Alt}_{1} \ldots \operatorname{Alt}_{k r}\left(\prod _ { i = 1 } ^ { m } f _ { 0 } \left(\operatorname{ad}\left(x_{11 i}^{v^{i-1}}\right), \ldots, \operatorname{ad}\left(x_{1 \ell i}^{v^{i-1}}\right) ;\right.\right.\right.\right.\right. \\
\left.\left.\left.\left.\ldots ; \operatorname{ad}\left(x_{k r, 1, i}^{v^{i-1}}\right), \ldots, \operatorname{ad}\left(x_{k r, \ell, i}^{v^{i-1}}\right)\right)\right) z\right] \ldots\right] \notin \operatorname{Id}^{H_{m^{2}}(\zeta)}(L) .
\end{array}
$$

Now we notice that $\operatorname{deg} f=n$. If we rename the variables of $f$ to $x_{1}, x_{2}, \ldots, x_{n}$, then $f$ satisfies all the conditions of the lemma.

Proof of Theorem 7.1. If $L$ is semisimple, then the assertion of the theorem is a consequence of [11, Example 10].

Suppose $L$ is not semisimple. By [11, Lemma 1], we still have the upper bound ${c_{n}}_{m^{2}(\zeta)}^{\left.H^{(}\right)}(L) \leqslant$ $(\operatorname{dim} L)^{n+1}$.

Let $r$ be the number from Lemma 7.3. Let $\ell:=\frac{\operatorname{dim} L}{m}$ and $k:=\left[\frac{n-1}{\ell m r}\right]$. We claim that for every $n \in \mathbb{N}$ there exists $\lambda \vdash n, m\left(L, H_{m^{2}}(\zeta), \lambda\right) \neq 0$, such that $\lambda_{i} \geqslant k r$ for all $1 \leqslant i \leqslant \ell m$. Consider the polynomial $f$ from Lemma 7.3 . It is sufficient to prove that $e_{T_{\lambda}}^{*} f \notin \operatorname{Id}^{H_{m^{2}}(\zeta)}(L)$ for some tableau $T_{\lambda}$ of the desired shape $\lambda$. It is known that $F S_{n}=\bigoplus_{\lambda, T_{\lambda}} F S_{n} e_{T_{\lambda}}^{*}$ where the summation runs over the set of all standard tableaux $T_{\lambda}, \lambda \vdash n$. Thus

$$
F S_{n} f=\sum_{\lambda, T_{\lambda}} F S_{n} e_{T_{\lambda}}^{*} f \nsubseteq \operatorname{Id}^{H_{m^{2}}(\zeta)}(L)
$$

and $e_{T_{\lambda}}^{*} f \notin \operatorname{Id}^{H_{m^{2}}(\zeta)}(L)$ for some $\lambda \vdash n$. We claim that $\lambda$ is of the desired shape. It is sufficient to prove that $\lambda_{\ell m} \geqslant k r$, since $\lambda_{i} \geqslant \lambda_{\ell m}$ for every $1 \leqslant i \leqslant \ell m$. Each row of $T_{\lambda}$ includes numbers of no more than one variable from each $X_{i}$, since $e_{T_{\lambda}}^{*}=b_{T_{\lambda}} a_{T_{\lambda}}$ and $a_{T_{\lambda}}$ is symmetrizing the variables of each row. Thus $\sum_{i=1}^{\ell m-1} \lambda_{i} \leqslant k r(\ell m-1)+(n-k \ell m r)=n-k r$. Lemma 7.2 implies that if $\lambda \vdash n$ and $\lambda_{\ell m+1}>0$, then $m\left(L, H_{m^{2}}(\zeta), \lambda\right)=0$. Therefore $\lambda_{\ell m} \geqslant k r$.

The Young diagram $D_{\lambda}$ contains the rectangular subdiagram $D_{\mu}, \mu=(\underbrace{k r, \ldots, k r}_{\ell m})$. The branching rule for $S_{n}$ implies that if we consider the restriction of $S_{n}$-action on $M(\lambda)$ to $S_{n-1}$, then $M(\lambda)$ becomes the direct sum of all non-isomorphic $F S_{n-1}$-modules $M(\nu), \nu \vdash$ $(n-1)$, where each $D_{\nu}$ is obtained from $D_{\lambda}$ by deleting one box. In particular, $\operatorname{dim} M(\nu) \leqslant$ $\operatorname{dim} M(\lambda)$. Applying the rule $(n-k \ell m r)$ times, we obtain $\operatorname{dim} M(\mu) \leqslant \operatorname{dim} M(\lambda)$. By the hook formula,

$$
\operatorname{dim} M(\mu)=\frac{(k \ell m r) !}{\prod_{i, j} h_{i j}}
$$

where $h_{i j}$ is the length of the hook with edge in $(i, j)$. By Stirling formula,

$$
\begin{gathered}
c_{n}^{H_{m^{2}}(\zeta)}(L) \geqslant \operatorname{dim} M(\lambda) \geqslant \operatorname{dim} M(\mu) \geqslant \frac{(k \ell m r) !}{((k r+\ell m) !)^{\ell m}} \sim \\
\frac{\sqrt{2 \pi(k \ell m r)}\left(\frac{k \ell m r}{e}\right)^{k \ell m r}}{\left(\sqrt{2 \pi(k r+\ell m)}\left(\frac{k r+\ell m}{e}\right)^{k r+\ell m}\right)^{\ell m}} \sim C_{1} k^{r_{1}}(\ell m)^{k \ell m r}
\end{gathered}
$$

for some constants $C_{1}>0, r_{1} \in \mathbb{Q}$, as $k \rightarrow \infty$. (We write $f \sim g$ if $\lim \frac{f}{g}=1$.) Since $k=\left[\frac{n-1}{\ell m r}\right]$, this gives the lower bound. 


\section{REFERENCES}

[1] Bakhturin, Yu. A. Identical relations in Lie algebras. VNU Science Press, Utrecht, 1987.

[2] Bakhturin, Yu. A., Zaicev, M. V., Sehgal, S. K. Finite-dimensional simple graded algebras. Sb. Math. 199:7-8 (2008), 965-983.

[3] Dăscălescu, S., Năstăsescu, C., Raianu, Ş. Hopf algebras: an introduction. New York, Marcel Dekker, Inc., 2001.

[4] Drensky, V.S. Free algebras and PI-algebras: graduate course in algebra. Singapore, Springer-Verlag, 2000.

[5] Elduque, A., Kochetov, M. V. Gradings on simple Lie algebras. AMS Mathematical Surveys and Monographs 189, Providence, R.I., 2013.

[6] Etingof, P., Ostrik, V. Finite tensor categories. Moscow Math. J., 4:3 (2004), 627-654.

[7] Giambruno, A., Zaicev, M. V. Polynomial identities and asymptotic methods. AMS Mathematical Surveys and Monographs 122, Providence, R.I., 2005.

[8] Giambruno, A., Zaicev, M. V. Exponential codimension growth of P.I. algebras: an exact estimate, Adv. Math., 142 (1999), 221-243.

[9] Gordienko, A.S. Graded polynomial identities, group actions, and exponential growth of Lie algebras. J. Algebra, 367 (2012), 26-53.

[10] Gordienko, A.S. Structure of $H$-(co)module Lie algebras. J. Lie Theory, 23:3 (2013), 669-689.

[11] Gordienko, A.S. Amitsur's conjecture for polynomial $H$-identities of $H$-module Lie algebras. Tran. Amer. Math. Soc., 367:1 (2015), 313-354.

[12] Gordienko, A. S. On a formula for the PI-exponent of Lie algebras. J. Alg. Appl., 13:1 (2013), 1350069-1 - 1350069-18.

[13] Gordienko, A. S. Algebras simple with respect to a Sweedler's algebra action. J. Alg. Appl., 14:1 (2015), 1450077-1 - 1450077-15.

[14] Gordienko, A. S. Algebras simple with respect to a Taft algebra action. J. Pure and Appl. Alg., 219 (2015), 3279-3291.

[15] Goto, M., Grosshans, F. Semisimple Lie algebras. Marcel Dekker, New York and Basel, 1978.

[16] Jacobson, N. Lie algebras. New York-London, Interscience Publishers, 1962.

[17] Kinser, R., Walton, C. Actions of some pointed Hopf algebras on path algebras of quivers. Algebra and Number Theory, 10:1 (2016), 117-154.

[18] Linchenko, V. Identities of Lie algebras with actions of Hopf algebras. Communications in Algebra, 25:10 (1997), 3179-3187.

[19] Mishchenko, S. P., Zaicev M. V. An example of a variety of Lie algebras with a fractional exponent. J. Math. Sci. (New York), 93:6 (1999), 977-982.

[20] Mishchenko, S.P., Verevkin, A.B., Zaitsev, M.V. A sufficient condition for coincidence of lower and upper exponents of the variety of linear algebras. Mosc. Univ. Math. Bull., 66:2 (2011), 86-89.

[21] Montgomery, S. Hopf algebras and their actions on rings. CBMS Lecture Notes 82, Amer. Math. Soc., Providence, RI, 1993.

[22] Montgomery, S., Schneider, H.-J. Skew derivations of finite-dimensional algebras and actions of the double of the Taft Hopf algebra. Tsukuba J. Math., 25:2 (2001), 337-358.

[23] Năstăsescu, C., Van Oystaeyen, F. Methods of graded rings. Lecture Notes in Mathematics 1836, Springer-Verlag, Berlin-Heidelberg, 2004.

[24] Razmyslov, Yu. P. Identities of algebras and their representations. Transl. Math. Monogr. 138, Amer. Math. Soc., Providence, RI, 1994.

[25] Sweedler, M. Hopf algebras. W.A. Benjamin, inc., New York, 1969.

[26] Taft, E. J. Invariant Wedderburn factors. Illinois J. Math., 1 (1957), 565-573.

[27] Volichenko, I. B. Varieties of Lie algebras with identity $\left[\left[X_{1}, X_{2}, X_{3}\right],\left[X_{4}, X_{5}, X_{6}\right]\right]=0$ over a field of characteristic zero. Sibirsk. Mat. Zh., 25:3 (1984), 40-54. (In Russian.)

[28] Zaitsev, M. V. Integrality of exponents of growth of identities of finite-dimensional Lie algebras. Izv. Math., 66 (2002), 463-487.

Vrije Universiteit Brussel, Belgium

E-mail address: alexey.gordienko@vub.ac.be 